\title{
Topological aspects of three-dimensional wakes behind rotary oscillating cylinders
}

\author{
By PHILIPPE PONCET \\ Laboratoire MIP, Dept GMM, INSA, 135 avenue de Rangueil, 31077 Toulouse Cedex 4, France
}

(Received february 6th 2002 and in revised form april 2003)

The development of a three-dimensional viscous incompressible flow generated behind an infinitely long circular cylinder, impulsively started into rectilinear motion and rotationally oscillating, is studied computationally. The numerical scheme, an hybrid vortex method, is used to integrate the velocity-vorticity formulation of the Navier-Stokes equations. The Reynolds number considered is $R e=400$, which is moderate though beyond the critical values $R e_{2} \simeq 190$ and $R e_{2}^{\prime} \simeq 260$ for which the flow becomes spontaneously three-dimensional. The numerical method is explained and its main points are developped. This scheme is then applied to solve a few two-dimensional problems, both in order to validate the method and to compute a nominal twodimensional flow, required to measure the impact of three-dimensionality. The three-dimensional flow past a steady cylinder is also compared to benchmark simulations. Once the flow has become fully three-dimensional, beyond transient regime and saturation of instabilities, the cylinder begins a rotary oscillation around its axis. Two kinds of rotations are considered, either at constant amplitude and several frequencies, or at constant frequency and various amplitudes. When amplitude and frequency are high enough, the whole flow comes back to its two-dimensional state. This result gives a justification for two-dimensional computations present in the literature related to rotating cylinders. For the first super-harmonic frequency of the flow, a parametric study is realized in order to get the impact of the amplitude on the topology of the flow. A bifurcation is clearly identified. Eventually, the mechanisms involved in the return to a two-dimensional state are explained : the interaction between transverse instabilities and von Kármán alleys is quantified by means of a correlation analysis.

\section{Introduction and related work}

The description of the wake behind bluff bodies in a viscous flow is of fundamental importance in many engineering and scientific fields. The canonical case of the circular cylinder has been reported in numerous works, concerning experimentations as well as numerical investigations, in the simplified case of a plan or in the physical three-dimensional space. The interest of body rotation is mainly motivated by the possibility of modifying the wake generated downstream and the forces created by the wake on this body. Indeed, such studies throw light on mechanisms that reduce reduce drag forces, which is a key point in aeronautics and other fields of application, described for example in Sümer \& Fredsøe (1997).

Most of prior works concerned either steady cylinder in three dimensions, or rotating cylinders in two dimensions, mainly due to restrictive Courant-Friedrich-Levy conditions and computational cost. Early three-dimensional measures, for a steady cylinder, are reported in Wieselsberger (1922), though the 3D cylinder has been a field of active research during the $90 \mathrm{~s}$.

For incompressible wakes generated by a circular cylinder, two of the governing non dimensional parameters are the Reynolds number $R e=U_{\infty} D / \nu$ where $D$ is the diameter of the 
cylinder, $U_{\infty}$ the free stream velocity and $\nu$ the kinematic viscosity, and the Strouhal number $S_{t}=f D / U_{\infty}$, where $f=1 / T, T$ being the natural period of the flow.

It is now well known that the wake of a circular cylinder becomes spontaneously threedimensional when $R e \geqslant R e_{2}=190$. The instabilities and non-spanwise structures of the flow have been exhibited experimentally by Williamson (1988), and later numerically by Tomboulides et al. (1992). Several contributors, as Karniadakis \& Triantafyllou (1992), Beaudan \& Moin (1994), Thompson et al. (1994, 1996), Persillon \& Braza (1998) or Kravchenko et al. (1999), have since studied the transition to turbulence and other aspects of these flows. Several diagnostics of the 3D flows have been computed, by Henderson (1995a) and Mittal (1996), in order to provide an analysis of $3 \mathrm{D}$ effects and comparison with experimental results such as Williamson (1996). Higher Reynolds numbers have been also considered in latest works, for example Mittal (1996) or Jordan (1997), who have considered sub-grid models.

Furthermore, it has been shown in Barkley \& Henderson (1996) that two modes of transition, commonly called Mode $A$ and Mode $B$, occur respectively at $R e_{2} \simeq 189$ and $R e_{2}^{\prime} \simeq 259$. Consequently, realistic numerical simulations have to be performed in 3D when $R e \geqslant 190$.

When the cylinder is moving in steady or oscillating rotation, or oscillating in translation (i.e. vibration), the condition of linear stability implies that time steps have to be very small (hence the usual restriction to two-dimensional computations). The first $2 \mathrm{D}$ numerical simulations of a more complex motion than a uniform translation have dealt with the steadily rotating cylinder, initially with Coutanceau \& Ménard (1985) and Badr \& Dennis (1985), followed by Chang \& Chern (1991) and Chen et al. (1993) among others. Badr et al. (1990) joins both experiments and numerical investigations in the range $10^{3} \leqslant R e \leqslant 10^{4}$. Oscillating flows, that is to say in pure oscillating translation, unlike uniform or accelerated motions, have been and are still a huge field of interest in naval research. Properties of these flows have been studied for wide ranges of Keulegan-Carpenter numbers by many contributors, for example Bearman (1997). On the other hand, studies on cylinders oscillating freely under crosswise translation and undergoing uniform rectilinear motion have been performed during the last years, both numerically by Blackburn \& Henderson (1999) and experimentally by Govardhan \& Williamson (2000). The same kind of problem with forced oscillations is developed in Dütch et al. (1998), who joined numerical simulation and experimentation.

The motion considered in the present paper is a uniform rectilinear motion with rotational oscillation. This type of motion has been experimentally studied for low Reynolds numbers by Taneda (1978), who has provided snapshots of streamlines, and by Tokumaru \& Dimotakis (1991) at $R e=15000$. Numerical simulations corresponding to this case have been lately performed by Lu \& Sato (1996), Chou (1997) and Baek \& Sung (1998). Accurate numerical simulations can be found in the recent papers by Dennis et al. (2000) and He et al. (2000), which are considered in the present paper as benchmarks for diagnostics. When rotations of higher amplitude are considered, diagnostics can be compared to the two-dimensional study by Milano et al. (2000) and Milano \& Koumoutsakos (2002).

So far all the numerical simulations for rotating cylinders seem to have focused on 2D geometries, probably due to the computational cost of 3D simulations and available numerical tools. In order to be realistic, for long time scale and for Reynolds numbers above 190, numerical simulations should take into account the three-dimensionality of the flow. In order to perform such numerical simulations, a robust numerical method has to be used, i.e. which still converges when used with large time steps, in order to reach large time scales.

The goal of the present paper is to describe $3 \mathrm{D}$ aspects of flows around a rotating cylinder, infinitely long. This cylinder is steady until the flow is fully three-dimensional, at $R e=400$. Then the cylinder begins to rotate, with an angular position given by the formula

$$
\theta(t)=-A \cos \left(\pi S_{F} t\right)
$$


with $S_{F}$ being the forced Strouhal number and $A$ the peak angular position, called amplitude. Consequently, the tangential velocity on the body is given by $V=R \dot{\theta}=A \pi S_{F} R \sin \left(\pi S_{F} t\right)$. The ratio $S_{F} / S_{t}$ will be chosen among integers in order to consider only harmonic and superharmonic frequencies of the flow. This avoids to focus on locking phenomena and competition between different frequencies.

The main result presented herein is that the flow comes back to a two-dimensional state in the neighbourhood of the body for high amplitudes. The question of the critical amplitude that leads to a $2 \mathrm{D}$ flow is also considered in the case for which $S_{F} / S_{t}=2$.

Furthermore, fast rotations can lead to a substantial drop of the drag coefficient. This fact has been exhibited experimentally in Tokumaru \& Dimotakis (1991) (at $R e=1.510^{4}$ ) and numerically in Milano et al. (2000). For medium-range frequencies of rotation, one can refer to Dennis et al. (2000) and He et al. (2000).

An outline of the present paper is as follows. The numerical method is first described in section 2, and a few two-dimensional diagnostics are computed in section 3, in order to provide a validation the numerical scheme. The present results are compared to $2 \mathrm{D}$ computations by Henderson (1995b), Henderson (1997), Utnes (1997), Blackburn \& Henderson (1999), Dennis et al. (2000) and He et al. (2000). Section 4 shows the spontaneous development of threedimensionality. For $R e=400 \geqslant R e_{2}^{\prime}=260$, mode $\mathrm{B}$ is identified by means of spectrum analysis, and diagnostics are compared to Barkley \& Henderson (1996), Henderson (1995a) and Wieselsberger (1922). The development of three-dimensionality is shown to be exponential. Section 5 shows the effect of a high amplitude of rotation $(A=\pi / 2)$ for a few frequencies, and exhibits representative phenomena due to the body rotation. The effect of the amplitude on the topology of the flow is then discussed in section 6 for $S_{F} / S_{t}=2$. Finally, in section 7, the mechanism leading to a two-dimensional flow is identified and discussed in the case $S_{F} / S_{t}=2$ and $A=\pi / 2$, and a correlation analysis is provided.

\section{The three-dimensional Navier-Stokes equations}

The present numerical method is a deterministic hybrid Vortex-In-Cell method (particles carry vorticity, see Cottet \& Koumoutsakos (2000) for general overview). Fields which are solutions of Poisson or Helmholtz equations are computed on a cylindrical grid instead of considering a pure lagrangian method. Recent developments in these methods for both two- and three-dimensional flow computations are described in Cottet \& Poncet (2003) and Cottet \& Poncet (2002), based on preliminary results presented in Cottet et al. (2002), Koumoutsakos \& Leonard (1995) and Ould-Sahili et al. (2000) for wake computations.

Let us consider the three-dimensional Navier-Stokes equations in their fully non-linear velocity-vorticity formulation

$$
\frac{\partial \omega}{\partial t}+\boldsymbol{u} \cdot \nabla \boldsymbol{\omega}-\boldsymbol{\omega} \cdot \nabla \boldsymbol{u}-\nu \nabla^{2} \boldsymbol{\omega}=0
$$

where $\boldsymbol{\nabla}$ is a gradient operator, $\nu$ is the kinematic viscosity, $\boldsymbol{\omega}$ is the vorticity field and $\boldsymbol{u}$ is the velocity field, satisfying

$$
\boldsymbol{\nabla} \cdot \boldsymbol{u}=0, \quad \boldsymbol{\nabla} \times \boldsymbol{u}=\boldsymbol{\omega} .
$$

thus $\boldsymbol{\nabla} \cdot \boldsymbol{\omega}=0$. One also considers a smooth three-dimensional body $\mathcal{B}$, which is an infinitely long circular cylinder. On this cylinder there is the no-slip boundary condition

$$
\boldsymbol{u}=V_{\text {slip }} \widehat{\boldsymbol{e}}_{\theta}
$$

where $V_{\text {slip }}$ is the tangential body velocity. If the cylinder is not rotating, then $V_{\text {slip }}=0$. 
All along this paper, the far field condition is

$$
\boldsymbol{u}(x) \rightarrow \boldsymbol{U}_{\infty} \text { as }|x| \rightarrow \infty
$$

and since the cylinder is infinitely long, all functions are periodic in the spanwise direction. This period is denoted by $L$ from now on. In order to simplify notations, these two last boundary conditions will no longer be written.

The basis is written $\left(\widehat{\boldsymbol{e}}_{x}, \widehat{\boldsymbol{e}}_{y}, \widehat{\boldsymbol{e}}_{z}\right)$ in Cartesian coordinates and $\left(\widehat{\boldsymbol{e}}_{r}, \widehat{\boldsymbol{e}}_{\theta}, \widehat{\boldsymbol{e}}_{z}\right)$ in cylindrical coordinates (see figure 1). The vector $\widehat{\boldsymbol{e}}_{x}$ is the streamwise direction and $\widehat{\boldsymbol{e}}_{y}$ the crosswise direction. All along this paper, the body $\mathcal{B}$ is a circular cylinder, whose axis is the spanwise direction $\widehat{\boldsymbol{e}}_{z}$. The computational domain is then $\Omega=\mathbb{R}^{3} \backslash \overline{\mathcal{B}}$.

\subsection{Particle methods}

In order to solve numerically the Navier-Stokes equations, one considers a Lagrangian "Particlein-Cell" method. This method, described below, is based on an hybrid formulation of vortex methods.

Indeed, particles carry cells of vorticity, and velocity reconstruction uses Helmoholtz and Poisson equations via back and forth interpolations on a grid, instead of a classical approach using multipole methods or stochastic methods as Monté-Carlo. This leads to a gain of accuracy (compared to Monté-Carlo methods) and speed (compared to the multipole approach, see Cottet \& Poncet (2002)).

Let the particles be indexed by $p=1 \ldots n$. A particle is described by its position $x_{p}(t)$, its vorticity $\omega_{p}(t)$, and its volume $v_{p}$. This volume does not depend on time due to the incompressibility. Thus one considers measure solutions such as

$$
\boldsymbol{\omega}(t)=\sum_{p=1}^{n} \boldsymbol{\omega}_{p}(t) \delta_{\boldsymbol{x}_{p}(t)} v_{p}
$$

where $\delta_{\boldsymbol{x}_{p}}$ is the Dirac function at $\boldsymbol{x}_{p}$.

The no-slip condition $(2.1 c)$ may be written in a scalar form, with a no-slip-through condition

$$
\boldsymbol{u} \cdot \widehat{\boldsymbol{e}}_{r}=0
$$

and the two tangential conditions

$$
\boldsymbol{u} \cdot \widehat{\boldsymbol{e}}_{\theta}=V_{\mathrm{slip}}, \boldsymbol{u} \cdot \widehat{\boldsymbol{e}}_{z}=0 .
$$

A fractional-step algorithm is then used to solve the Navier-Stokes equations $(2.1 a-c)$ with the measure solution (2.2). Let $\omega_{0}$ be a measure solution at time $t_{0}$.

The first step is pure convection, which ensures the no-slip-through condition $(2.3 a)$ :

$$
\left\{\begin{aligned}
\frac{d \boldsymbol{\omega}_{p}}{d t} & =[\boldsymbol{\nabla} \cdot(\boldsymbol{u} \otimes \boldsymbol{\omega})]_{\boldsymbol{x}_{p}(t)} \\
\frac{d \boldsymbol{x}_{p}}{d t} & =u\left(\boldsymbol{x}_{p}(t)\right)
\end{aligned}\right.
$$

for $t \in\left[t_{0}, t_{0}+\delta t\right]$, where fields $\boldsymbol{u}$ and $\boldsymbol{\omega}$ satisfy

$$
\begin{cases}\boldsymbol{\nabla} \times \boldsymbol{u}=\boldsymbol{\omega}, \boldsymbol{\nabla} \cdot \boldsymbol{u}=0 & \text { in } \Omega \times\left[t_{0}, t_{0}+\delta t\right] \\ \boldsymbol{\omega}\left(t_{0}\right)=\omega_{0} & \text { on } \Omega \\ \boldsymbol{u} \cdot \widehat{\boldsymbol{e}}_{r}=0 & \text { on } \partial \Omega \times\left[t_{0}, t_{0}+\delta t\right]\end{cases}
$$

The stretching tensor $\boldsymbol{\nabla} \cdot(\boldsymbol{u} \otimes \boldsymbol{\omega})$ is equal to $\boldsymbol{\omega} \cdot \boldsymbol{\nabla} \boldsymbol{u}$ when $\boldsymbol{\nabla} \cdot \boldsymbol{\omega}=0$. This last tensor makes the 
numerical scheme more stable when the vorticity is not algebrically divergence-free (see Cottet (1996)). It remains to treat the diffusion and the tangential no-slip condition (2.3b). Moreover, the main difficulty of step (2.4) is the computation of the velocity field, which is discussed in the following subsection.

\subsection{Velocity computation and Grid-Particle coupling}

The initial data of step (2.4) is the vorticity field $\omega_{0}$. This field contains the position, the pointwise vorticity and the volume of all particles. Thus step (2.4) is a classical dynamical system as soon as the velocity field is known. This velocity must satisfy

$$
\left\{\begin{array}{cc}
\boldsymbol{\nabla} \times \boldsymbol{u}=\boldsymbol{\omega} & \text { on } \Omega \\
\boldsymbol{\nabla} \cdot \boldsymbol{u}=0 & \text { on } \Omega \\
\boldsymbol{u} \cdot \widehat{\boldsymbol{e}}_{r}=0 & \text { on } \partial \Omega
\end{array}\right.
$$

The usual deterministic approach of vortex methods is to compute this velocity field with the Biot-Savart laws :

$$
\boldsymbol{u}\left(x_{q}\right)=\left[K_{\varepsilon} * \boldsymbol{\omega}\right]\left(x_{q}\right)=\int_{\Omega} K_{\varepsilon}\left(x_{q}-x\right) \boldsymbol{\omega}(x) \mathrm{d} x=\sum_{x_{p}} K_{\varepsilon}\left(x_{q}-x_{p}\right) \boldsymbol{\omega}\left(x_{p}\right) v_{p}
$$

given the particle description (2.2) of the vorticiy. Such an approach requires $n^{2}$ evaluations of the mollified Green kernel $K_{\varepsilon}, n$ being the number of particles. This computation is not yet technically possible for three-dimensional computations involving millions of particles (see Cottet \& Poncet (2003) and Cottet \& Poncet (2002)) in time-dependent simulations, despite recent advances in multipole methods by Strickland et al. (1999), Plouhmans \& Winckelmans (2000) or Lindsay \& Krasny (2001).

An alternative to compute the velocity field is to use a stream function $\psi$. For any divergencefree vorticity field and at any time $t$, the link between the stream function and vorticity is

$$
-\nabla^{2} \psi=\omega
$$

with arbitrary boundary conditions which imply $\boldsymbol{\nabla} \cdot \boldsymbol{\psi}=0$ (thus $\boldsymbol{\psi}$ is globally divergence-free). One also considers an harmonic potential $\phi$, that is to say

$$
\nabla^{2} \phi=0
$$

which satisfies

$$
\frac{\partial \phi}{\partial n}=\nabla \times \boldsymbol{\psi} \cdot \widehat{\boldsymbol{e}}_{r}
$$

on the boundary $\partial \Omega$. Eventually the velocity is computed by the Helmholtz decomposition

$$
u=\nabla \times \psi-\nabla \phi
$$

and verifies equations (2.6).

In order to solve the Poisson equations $(2.7 a, b)$, the vorticity is interpolated onto a grid. The velocity is computed on this grid and interpolated back to particles. Such a method is called hybrid due to the fact that it uses both particles and grids. The interpolation is a third order tensor product formula (see Monaghan (1985)), except near the body where one-sided formulas are prefered. Details of this strategy of interpolation can be found in Cottet \& Poncet (2003).

\subsection{Diffusion and boundary layer}

Our approach is an extension to 3D of the method used by Koumoutsakos et al. (1994) to study 2D diffusion and vorticity boundary conditions. Diffusion in the fluid, the second step of the 
fractional-step algorithm, is performed through vorticity exchange among particles. The no-slip condition (third step) is enforced by means of a vorticity flux at the boundary.

More precisely, let $\omega_{1}$ be the final value at $t=t_{0}+\delta t$ of the first step 2.4. The vorticity field on the boundary can be written in cylindrical coordinates $\boldsymbol{\omega}=\left(\omega_{r}, \omega_{\theta}, \omega_{z}\right)$. The diffusion is given by the heat equation :

$$
\begin{cases}\frac{\partial \boldsymbol{\omega}}{\partial t}=\nu \nabla^{2} \boldsymbol{\omega} & \text { in } \Omega \times\left[t_{0}, t_{0}+\delta t\right] \\ \frac{\partial \omega_{z}}{\partial n}=0 & \text { on } \partial \Omega \times\left[t_{0}, t_{0}+\delta t\right] \\ \kappa \omega_{\theta}+\frac{\partial \omega_{\theta}}{\partial n}=0 & \text { on } \partial \Omega \times\left[t_{0}, t_{0}+\delta t\right] \\ \omega_{r}=0 & \text { on } \partial \Omega \times\left[t_{0}, t_{0}+\delta t\right] \\ \boldsymbol{\omega}\left(t_{0}\right)=\omega_{1} & \text { on } \Omega\end{cases}
$$

where $\kappa=1 / R$ is the curvature of the cylinder. For a small viscosity $\nu$, i.e. for a Reynolds number high enough, this equation can be solved with an explicit scheme, a "Particle Strength Exchange" algorithm, developped in Degond \& Mas-Gallic (1989).

Let $\omega_{2}$ be the solution of (2.8). Furthermore, for any $t$ between $t_{0}$ and $t_{o}+\delta t$, the velocity produced by the solution of (2.8) is written $\boldsymbol{u}_{\mathrm{spu}}(t)$ and is commonly called spurious velocity. This velocity $\boldsymbol{u}_{\mathrm{spu}}(t)$ is computed by solving equation (2.6).

The no-slip condition $(2.3 b)$ is enforced by means of the boundary layer. This viscous boundary layer is the step 3 of the fractional-step algorithm, another heat equation :

$$
\begin{cases}\frac{\partial \boldsymbol{\omega}}{\partial t}=\nu \nabla^{2} \boldsymbol{\omega} & \text { on } \Omega \times\left[t_{0}, t_{0}+\delta t\right] \\ \nu \frac{\partial \omega_{z}}{\partial n}=-\frac{\partial \boldsymbol{u}_{\mathrm{spu}}}{\partial t} \cdot \widehat{\boldsymbol{e}}_{\theta}-\frac{\partial V_{\mathrm{slip}}}{\partial t} & \text { on } \partial \Omega \times\left[t_{0}, t_{0}+\delta t\right] \\ \nu \kappa \omega_{\theta}+\frac{\partial \omega_{\theta}}{\partial n}=\frac{\partial \boldsymbol{u}_{\mathrm{spu}}}{\partial t} \cdot \widehat{\boldsymbol{e}}_{z} & \text { on } \partial \Omega \times\left[t_{0}, t_{0}+\delta t\right] \\ \omega_{r}=0 & \text { on } \partial \Omega \times\left[t_{0}, t_{0}+\delta t\right] \\ \boldsymbol{\omega}\left(t_{0}\right)=0 & \text { on } \Omega\end{cases}
$$

where $V_{\text {slip }}=R \dot{\theta}$ is the velocity on the surface of the body due to the rotation. The Robin 
boundary conditions enforce $\partial_{t} \boldsymbol{\nabla} \cdot \boldsymbol{\omega}=0$. Indeed, one has

$$
\begin{aligned}
\frac{\partial}{\partial r}(\boldsymbol{\nabla} \cdot \boldsymbol{\omega}) & =\left(\nabla^{2} \omega_{r}-\frac{\omega_{r}}{r^{2}}-\frac{2}{r^{2}} \frac{\partial \omega_{\theta}}{\partial \theta}\right)+\frac{1}{r^{2}} \frac{\partial \omega_{\theta}}{\partial \theta}+\frac{1}{r} \frac{\partial}{\partial \theta} \frac{\partial \omega_{\theta}}{\partial r}+\frac{\partial}{\partial z} \frac{\partial \omega_{z}}{\partial r} \\
& =\left(\nabla^{2} \boldsymbol{\omega}\right) \cdot \vec{e}_{r}+\frac{1}{r} \frac{\partial}{\partial \theta}\left(\frac{1}{r} \omega_{\theta}\right)+\frac{1}{r} \frac{\partial}{\partial \theta} \frac{\partial \omega_{\theta}}{\partial r}+\frac{\partial}{\partial z} \frac{\partial \omega_{z}}{\partial r} \\
& =\frac{1}{\nu} \frac{\partial \omega_{r}}{\partial t}+\frac{1}{r} \frac{\partial}{\partial \theta}\left(\kappa \omega_{\theta}+\frac{\partial \omega_{\theta}}{\partial r}\right)+\frac{\partial}{\partial z} \frac{\partial \omega_{z}}{\partial r} \\
& =\frac{1}{\nu} \frac{\partial}{\partial t} \frac{\partial \boldsymbol{u}_{\mathrm{spu}} \cdot \widehat{\boldsymbol{e}}_{z}}{r \partial \theta}-\frac{1}{\nu} \frac{\partial}{\partial t} \frac{\partial \boldsymbol{u}_{\mathrm{spu}} \cdot \widehat{\boldsymbol{e}}_{\theta}}{\partial z} \\
& =\frac{1}{\nu} \frac{\partial}{\partial t}\left(\boldsymbol{\nabla} \times \boldsymbol{u}_{\mathrm{spu}} \cdot \widehat{\boldsymbol{e}}_{r}\right)=\frac{1}{\nu} \frac{\partial}{\partial t}\left(\boldsymbol{\omega}_{2} \cdot \widehat{\boldsymbol{e}}_{r}\right)=0
\end{aligned}
$$

Consequently, divergence satisfies the well-posed heat equation

$$
\begin{cases}\frac{\partial(\boldsymbol{\nabla} \cdot \boldsymbol{\omega})}{\partial t}-\nu \nabla^{2}(\boldsymbol{\nabla} \cdot \boldsymbol{\omega})=0 & \text { in } \Omega \times] t_{0}, t_{0}+\delta t[ \\ \boldsymbol{\nabla} \cdot \boldsymbol{\omega}\left(t_{0}\right)=0 & \text { on } \Omega \\ \frac{\partial}{\partial r}(\boldsymbol{\nabla} \cdot \boldsymbol{\omega})=0 & \text { on } \partial \Omega \times] t_{0}, t_{0}+\delta t[\end{cases}
$$

thus remains zero over time and space.

The solution of (2.9) can be computed in its integral form, see Friedmann (1964) and Koumoutsakos et al. (1994):

$$
\boldsymbol{\omega}(x, t)=\int_{t_{0}}^{t} \int_{\partial \Omega} G(x, t ; \xi, \tau) \boldsymbol{\mu}(\xi, \tau) \mathrm{d} \xi \mathrm{d} \tau
$$

where $G$ is the three-dimensional heat kernel, with $\tau<t$ :

$$
G(x, t ; \xi, \tau)=(4 \pi \nu(t-\tau))^{-3 / 2} \exp \left(-\frac{\|x-\xi\|^{2}}{4 \nu(t-\tau)}\right)
$$

and $\boldsymbol{\mu}$ a weighted field defined on the physical boundary. The construction of this weighted field is based on the first order development of the integral equation satisfied by $\boldsymbol{\mu}$, and does not create divergence as seen above (see Cottet \& Poncet (2003)). This technique is a very robust and flexible algorithm for boundary layer computation, and can be used in various situations involving tangential velocities (see control strategies in Poncet (2003), Cottet \& Poncet (2004) and Poncet (2004)).

\subsection{Conclusion related to the numerical scheme}

A fractional step algorithm (2.4)-(2.8)-(2.9) has been developped in order to approximate the solutions of the Navier-Stokes equations. The first step, given by equation (2.4), is a convection step. The second one is the diffusion step, given by the heat equation (2.8). The third and last step is the boundary layer computation, defined by equation (2.9). It enforces the no-slip conditions $(2.3 b)$. Moreover, particles are periodically remeshed, mainly using the third order kernel introduced by Monaghan (1985).

If we write respectively $\omega_{2}$ and $\omega_{3}$ the solutions of (2.8) and (2.9) at $t=t_{0}+\delta t$, then 
$\omega_{2}+\omega_{3}$ is a second order approximation of the solution of the Navier-Stokes equations (2.1ac) at $t=t_{0}+\delta t$.

The computational domain $\Omega$ used for three-dimensional simulations at $R e=400$ is an "Ogrid" defined by

$$
R \leqslant r \leqslant R+4 \pi R \text { and }-2 \pi R \leqslant z \leqslant 2 \pi R
$$

The discretisation is $256 \times 128 \times 128$ for the variables $r, \theta$ and $z$ respectively. The strong stability of the lagrangian method allows to use a non-dimensional time step $U_{\infty} \delta t / R=0.1$. The solver takes about 145 seconds per time step with the resolution above, which means about $35 \mu$ s per grid point on a DEC- $\alpha$ server.

\subsection{Mechanical diagnostics}

The non-dimensional time $t$ depends on the dimensional time $t^{\star}$ by the relation

$$
t=\frac{U_{\infty} t^{\star}}{R}
$$

From now on only the non-dimensional time $t$ will be used.

The Reynolds number is defined as

$$
\operatorname{Re}=\frac{U_{\infty} D}{\nu}
$$

and the non-dimensional frequency of the flow, that is to say the Strouhal number, is defined as

$$
S_{t}=\frac{f D}{U_{\infty}}
$$

where $f=1 / T$ is the natural frequency of the flow. The drag and lift coefficients are computed as in Koumoutsakos \& Leonard (1995). The friction coefficients are defined by :

$$
C_{D F}=-\frac{\nu}{U_{\infty}^{2} R L} \int_{\partial \Omega} \omega_{z} \sin \theta \mathrm{d} s, \quad C_{L F}=\frac{\nu}{U_{\infty}^{2} R L} \int_{\partial \Omega} \omega_{z} \cos \theta \mathrm{d} s,
$$

and the pressure coefficients by

$$
C_{D P}=-\frac{\nu}{U_{\infty}^{2} R L} \int_{\partial \Omega} \frac{\partial \omega_{z}}{\partial r} r \sin \theta \mathrm{d} s, \quad C_{L P}=\frac{\nu}{U_{\infty}^{2} R L} \int_{\partial \Omega} \frac{\partial \omega_{z}}{\partial r} r \cos \theta \mathrm{d} s .
$$

Thus the expression of the drag coefficient

$$
C_{D}=C_{D F}+C_{D P}
$$

and the lift coefficient

$$
C_{L}=C_{L F}+C_{L P}
$$

\section{Drag coefficients of two-dimensional flows}

In order to provide a validation of both the numerical scheme and study the behaviour of the nominal 2D flow, we perform several 2D simulations. Since these 2D computations exhibit the nominal state of forthcoming 3D simulations, the Reynolds number is chosen in the range between 200 and 1000 .

\subsection{Drag coefficient of a flow past a steady cylinder at various Reynolds numbers}

In this section, a few values of the drag and lift coefficients are computed in the case of a twodimensional flow.

The cylinder is impulsively started at $t=0^{+}$, and is slightly rotating during the early time with 


\begin{tabular}{|c|c|c|c|c|c|c|c|c|}
\hline$R e$ & & $\overline{C_{D}}$ & $\widehat{C_{L}}$ & $S_{t}$ & $R e$ & $\overline{C_{D}}$ & $\widehat{C_{L}}$ & $S_{t}$ \\
\hline 200 & 1.3389 & \pm 0.0015 & 0.70 & 0.199 & 200 & 1.3412 & $0.700^{\dagger}$ & $0.1972,0.196^{\dagger}$ \\
\hline 300 & 1.3820 & \pm 0.0031 & 0.96 & 0.211 & 300 & 1.3769 & - & 0.2113 \\
\hline 400 & 1.4080 & \pm 0.0043 & 1.08 & 0.2228 & 400 & 1.4142 & - & 0.2198 \\
\hline 500 & 1.4433 & \pm 0.0050 & 1.23 & 0.230 & 500 & 1.4449 & $1.19^{*}$ & 0.2254 \\
\hline 1000 & 1.5264 & \pm 0.0097 & 1.45 & 0.241 & 1000 & $1.5191^{\ddagger}$ & - & $0.2372,0.2392^{\ddagger}$ \\
\hline
\end{tabular}

TABLE 1. Diagnostics for Reynolds numbers between 200 and 2000. Left : Present computations. Right : Henderson (1995b) and Henderson (1997), except * Blackburn \& Henderson (1999), ${ }^{\dagger}$ Utnes (1997) and ${ }_{\ddagger}^{\ddagger}$ He et al. (2000). The error on $\overline{C_{D}}$ is the $90 \%$ confidence interval of the mean drag coefficient.

a tangential velocity $V_{\text {slip }}=\sin (\pi t / 2)$ while $t \leqslant 4$. This slight rotation is performed in order to break the crosswise symmetry of the flow. This triggers on the first von Kármán instability, which would appear later otherwise, thus a gain of computational time since this aspect of the flow is not the topic of the present study. For $t \geqslant 4$, the cylinder is steady, i.e. $V_{\text {slip }}=0$.

Figure 3 shows the drag and lift coefficient for various Reynolds numbers between 200 and 1000. Table 1 shows values of the mean value of the drag coefficient $C_{D}$, denoted $\overline{C_{D}}$, the mean of peak values of the absolute lift coefficient $\left|C_{L}\right|$, denoted $\widehat{C_{L}}$, and the Strouhal number $S_{t}$. On this table, one can read the values found by the present method, and compared to the results of Henderson (1995b) and Blackburn \& Henderson (1999). Note that Blackburn \& Henderson (1999) gives a range of $1.18-1.20$ for $\widehat{C_{L}}$, which emphases the sensitivity of this quantity. One can notice a quite good agreement between present values and the related works. In order to get such an accuracy, the computational domain has been radially extended up to $(1+8 \pi) R$ for these two-dimensional simulations.

\subsection{Drag coefficient of a flow past a rotationally oscillating cylinder}

This discussion on two-dimensional flows can be found under several forms in the existing literature. Note that Dennis et al. (2000) provides a lot of diagnostics on a rotary oscillating cylinder, especially on drag and lift coefficients.

The simulation begins exactly as described in the last section, i.e. the symmetry of the flow is broken by a slight rotation before $t \leqslant 4$, and then the cylinder stays steady. At $t=60$, the rotation is activated. Our formula of the tangential velocity on the body is

$$
V_{\text {slip }}=A \pi S_{F} R \sin \left(\pi S_{F} t\right)
$$

where $S_{F}$ is the forced Strouhal number, $A$ the rotation amplitude and $R$ the radius of the cylinder. The quantity $S_{F}$ is defined in the same spirit as the natural Strouhal number $S_{t}$ (see equation 2.10), that is to say

$$
S_{F}=\frac{f_{c} D}{U_{\infty}}
$$

where $f_{c}$ is the frequency of the cylinder rotation.

If a point on the cylinder is initially set at $\theta(0)=-A$, its location at time $t$ is given by

$$
\theta(t)=\theta(0)+\frac{1}{R} \int_{0}^{t} V_{\text {slip }}(u) \mathrm{d} u=-A \cos \left(\pi S_{F} t\right)
$$

since $V_{\text {slip }}=R \dot{\theta}$. This makes the formula (3.1) especially interesting.

The forced Strouhal number $S_{F}$ is chosen as a multiple of the natural Strouhal number $S_{t}$. Moreover, we will call high amplitude a rotation whose amplitude $A$ is $\pi / 2$.

It is known that a rotation whose amplitude and frequency are high enough (at least $S_{F}=$ 


$\begin{array}{cccccccc}S_{F} / S_{t} & 0 & 1.0 & 1.2 & 1.45 & 1.45 & 2.0 & 3.0 \\ A & - & \pi / 2 & \pi / 2 & \pi / 2 & 3 / \pi & \pi / 2 & \pi / 2 \\ V_{\max } & 0 & 1.135 & 1.362 & 1.646 & 1 & 2.270 & 3.405 \\ \overline{C_{D}} & 1.443 & 2.60 & 1.66 & 1.36 & 1.30 & 1.09 & 0.82\end{array}$

TABLE 2. Mean drag coefficients at $R e=500$ for various rotation parameters $\left(V_{\max }=A \pi S_{F}\right)$.

$2 S_{t}$ ) leads to a substantial drop of the drag coefficient (cf. numerical investigations of He et al. (2000), Milano et al. (2000), Dennis et al. (2000)). The drag coefficient may even decrease by a factor 2 for high Reynolds number, as stipulated in the experimental work by Tokumaru \& Dimotakis (1991) at $R e=15000$.

The table 2 shows the mean drag coefficients at $R e=500$ for a few forced Strouhal numbers from $S_{F}=S_{t}$ up to $S_{F}=3 S_{t}$, with $A=\pi / 2$. When $S_{F} \leqslant 1.45 S_{t}$, the mean drag decreases. Moreover, the drag coefficient decreases by $25 \%$ for $S_{F}=2 S_{t}$ and by $43 \%$ for $S_{F}=3 S_{t}$. A special case with $S_{F}=1 / 3$ and $A=3 / \pi$ has been performed in order to be compared with Dennis et al. (2000), whose cylinder is initially rotating. The present computation gives $\overline{C_{D}}=1.30$ which somehow disagrees with Dennis et al. (2000) who find a value close to 1.11. Given the wide range of values for $\overline{C_{D}}$ presented herein and in Dennis et al. (2000), He et al. (2000) and Milano et al. (2000), and given the sensitivity of the same quantity in Tokumaru \& Dimotakis (1991), the accuracy of the present values of $\overline{C_{D}}$ is questionable when the cylinder is rotating at high frequency. Nevertheless, present computations and those provided by He et al. (2000) are compatible and seem to be converged.

\section{Three-dimensional flow past a steady cylinder}

It is now well known that the wake of a circular cylinder becomes spontaneously threedimensional when $R e>190$. This fact is well investigated and analysed in Barkley \& Henderson (1996) for the linearized Navier-Stokes equations, or in the survey paper by Williamson (1996), for the experimental aspects. After this transition, the von Kármán alleys are no longer spanwise invariant. If $R e>260$, thinner structures appear as links between the alleys, sometimes referred as "fingers" of vorticity (cf. Jordan (1997)). These thin structures corresponds to the mode of instability called mode $B$, and Barkley \& Henderson (1996) show that their wavelength is $0.82 D$, and that they appear at $R e=259 \pm 2$.

Since the present computations are performed at $R e=400$, the mode B is the dominant mode of instability and we will focus our attention on its wavelength. One considers the Fourier transform of the velocity

$$
\widehat{\boldsymbol{u}}_{\beta}(x, y)=\int_{0}^{L} \boldsymbol{u}(x, y, z) e^{-2 i \pi \beta z / L} \mathrm{~d} z
$$

which corresponds to a non-dimensional wavelength $\lambda_{z} / D=L / \beta D$. The axial energy spectrum is defined by

$$
\left\|\widehat{\boldsymbol{u}}_{\beta}\right\|_{2}^{2}=\int_{\mathbb{R}^{2}}\left|\widehat{\boldsymbol{u}}_{\beta}(x, y)\right|^{2} \mathrm{~d} x \mathrm{~d} y
$$

with respect to the non-dimensional wavelength $\lambda_{z} / D$.

This spectrum is plotted on figure 5, which exhibits a strong resonance around $\lambda_{z} / D \simeq \pi / 8$, that is to say around 0.79 . Furthermore, near the transition at $R e_{2}^{\prime}=260$, wavelength of mode B has been predicted close to $\lambda_{z} / D=0.82$ by Barkley \& Henderson (1996). This wavelength can 
change as $R e$ increases since the domain of unstable wavelength becomes larger. One can even find several wavelengths of mode B instability for a same Reynolds number, depending on the streamwise position, when $R e>600$ (see Barkley \& Henderson (1996) and Persillon \& Braza (1998)). The mode B is the main mode of turbulence, at least until $R e=1900$ (see Unal \& Rockwell (1988)), where turbulence begins to govern the flow.

On the one hand, these properties justify the comparisons between flows at $R e=260$ and $R e=400$. On the other hand, this explains why the velocity spectrum $\beta \rightarrow\left\|\widehat{\boldsymbol{u}}_{\beta}\right\|_{2}^{2}$ plotted on figure 5 is exponential and does not exhibit a $-5 / 3$ power behaviour. Indeed, in the range $190 \leqslant$ $R e \leqslant 2000$, cylinder wakes are three-dimensional but not turbulent. Moreover, figure 5 shows the velocity spectra related to the development of the instabilities $(t=60)$, the saturation of these instabilities $(t=100)$, and when the three-dimensionality is fully developed (at $t=150$ ).

The evolution of the three-dimensionality inside the computational domain can be exhibited by means of the transverse enstrophy $Z^{\perp}=$ defined as follows :

$$
Z^{\perp}=\frac{1}{2}\left(\left\|\omega_{x}\right\|_{2}^{2}+\left\|\omega_{y}\right\|_{2}^{2}\right)=\frac{1}{2} \int_{\Omega} \omega_{x}^{2}(\xi) \mathrm{d} \xi+\frac{1}{2} \int_{\Omega} \omega_{y}^{2}(\xi) \mathrm{d} \xi
$$

which is plotted with respect to time on figure 6 . This gives the quantity of transverse vorticity (vorticity which is not spanwise), which means a measure of the three-dimensionality of the flow in the neighbourhood of the body. The figure 6 exhibits in more details the phenomenon shown on the spectra of figure $5:$ at $t \simeq 40$, the non-axial noise get structured and the flow enters in an exponential development of mode $\mathrm{B}$, i.e.

$$
\frac{1}{2}\left(\left\|\omega_{x}\right\|_{2}^{2}+\left\|\omega_{y}\right\|_{2}^{2}\right) \simeq a e^{t / 2.9}
$$

with $a=7.1410^{-12}$. At $t \simeq 110$, these instabilities saturate and the three-dimensionality is then fully developed. One may notice that this exponential behaviour is not noisy.

Moreover, the spectral profile $\mathfrak{P}$ is introduced in order to catch the spanwise structures for a given wavelength. This spectral profile is defined by

$$
\mathfrak{P}_{\lambda / D}(x, y)=\left|\widehat{\boldsymbol{u}}_{L / \lambda}(x, y)\right|^{2}
$$

Figure 7 shows this profile at the resonance wavelength, compared to the profile of Barkley \& Henderson (1996) found at $R e=260$. Once again the two results are in good agreement.

The effect of three-dimensionality on the drag coefficient $C_{D}$ is plotted on figure 10, as well as the friction coefficient $C_{D F}$, the 2D mean value at 1.41 from table 1 , coherent with Henderson (1995b), and eventually the value 1.19 , coherent with the $3 \mathrm{D}$ experimental results by Wieselsberger (1922) and Jordan \& Fromm (1972). Moreover, the friction coefficient $C_{D F}$ does not seem to be much affected by the strong three-dimensionality of the flow in the neighbourhood of the cylinder.

In addition of these diagnostics, one can also notice, on an more observational level, the high similarity between the present results and experimental snapshots provided by Williamson (1996). Indeed, a surface of isovorticity obtained beyond the transient is plotted on figure 8 and compared to an experimental picture by Williamson (1996). The similarity concerns the spacing between von Kármán alleys (due to the small dependency of the Strouhal number on the Reynolds number above $R e_{2}^{\prime}$ ), the topology of fingers of vorticity, the location and the size of knots, and local dislocations of spanwise alleys.

\section{3D flows past a rotating cylinder with high amplitude rotation}

This section focuses on flows behind a cylinder rotating by half a revolution, i.e. $A=\pi / 2$, with various rotation frequencies. The last section has shown that the three-dimensionality of the 
flow past a steady cylinder and its effects on diagnostics have been well resolved at $R e=400$. Figure 6 shows that the saturated regime is reached around $t \simeq 100$. In order to avoid any transient effect, one waits for $t=160$ before activating the rotation. Then the cylinder begins to oscillate around its axis according to formula (3.1).

In order to know the effect of the rotation on the topology of the flow, one plots several surfaces of isovorticity, at several levels and different times, for $S_{F}=S_{t}$ and $S_{F}=2 S_{t}$. This chronology is shown on figure 13, on which one can see the evolution of the three-dimensionality in the neighbourhood of the body by means of the transverse vorticity :

$$
\omega^{\perp}=\left(\omega_{x}^{2}+\omega_{y}^{2}\right)^{1 / 2}
$$

One can see on figure 13 that the three-dimensionality tends to disappear (see also Poncet (2002)). Indeed, only a small quantity of transverse vorticity remains at $t=240$ when $S_{F}=S_{t}$, i.e. when the rotation frequency is locked at the natural frequency of the flow $S_{t}=0.223$. Moreover, one can also notice that no visible three-dimensionality remains at $t=240$ at higher frequency $S_{F}=2 S_{t}$, i.e. when the rotation frequency is the first superharmonic of the nominal flow.

This phenomenon is quantified on figure 11 , which shows the transverse enstrophy $Z^{\perp}$, that is to say half the mean square of transverse vorticity over the computational domain. One can see that the superharmonic rotation makes the transverse enstrophy decrease down to $510^{-7}$, which corresponds to $210^{-13}$ per particle (and roughly half per grid point). Consequently we can say that the flow is totally two-dimensional in a large neighbourhood of the cylinder. Besides, when $S_{F}=S_{t}$, the transverse enstrophy tends to a mean value of 3.05 , which means that the global three-dimensionality is divided by 65 , i.e. decreases by $98.5 \%$.

Since the flow comes back to a two-dimensional state when $S_{F}=2 S_{t}$, the drag coefficient $C_{D}$ should be compared to the drag coefficient of the two-dimensional rotating cylinder. This comparison is shown on figure 12, where we can see that both the 2D and 3D computations lead to a mean drag coefficient value of 1.05. The nominal two-dimensional drag coefficient is 1.41 , and the 3D unforced drag coefficient is 1.19 : this means a drop of $12 \%$ from the $3 \mathrm{D}$ flow and $26 \%$ from the $2 \mathrm{D}$ flow.

\section{Effect of the amplitude on three-dimensionality}

In order to study the effect of the amplitude on the three-dimensionality of the flow, one has to consider a constant frequency. Indeed, for a given frequency, for example $S_{F}=2 S_{t}$, we have seen in last section that an amplitude of $A=\pi / 2$ makes the flow come back to its twodimensional state. This is not the same situation for $A=\pi / 5$, in which case the flow remains $3 \mathrm{D}$, as it can be seen on figure 14 .

The question addressed in this section is to find a critical value $A_{0}$ which makes the separation between 2D and 3D flows. This critical amplitude depends on the rotation frequency $S_{F}$ and on the Reynolds number : given the computational cost of these simulations, it is way too ambitious to consider a parametric study of the bifurcation.

Consequently, we focus on the case $R e=400$ and $S_{F}=2 S_{t}$, and search a small interval of values of $A$ for which the top of the interval leads to a 2D flow and the bottom of the interval leads to a 3D flow. The tool which allows to decide whether the flow is $2 \mathrm{D}$ or 3D in the neighbourhood $\Omega$ of the body is the same as in last section, i.e. the transverse enstrophy :

$$
Z^{\perp}=\frac{1}{2}\left(\left\|\omega_{x}\right\|_{2}^{2}+\left\|\omega_{y}\right\|_{2}^{2}\right)=\frac{1}{2} \int_{\Omega} \omega_{x}^{2}(\xi) \mathrm{d} \xi+\frac{1}{2} \int_{\Omega} \omega_{y}^{2}(\xi) \mathrm{d} \xi
$$

As a first approach, one can plot $Z^{\perp}$ for a wide range of amplitudes $A$. Figure 15 shows 
these curves for amplitudes from $A=\pi / 5$ up to $A=\pi / 2$ with a logarithmic scale. On this figure, three curves, corresponding to $A=\pi / 2, A=2 \pi / 5$ and $A=\pi / 3$, lead clearly to a two-dimensional flow. The two curves above, i.e. $A=\pi / 3.5$ and $A=\pi / 3.75$, do not seem to decrease down to zero. Nevertheless, between the activation of the rotation at $t_{c}=160$ and $t=300$, the mean transverse enstrophy has been divided by more than 400 , thus one can consider that this flow is two-dimensional. In order to clarify this property, the same quantity is plotted without logarithmic scale on figure 16 . One checks that $A=2 \pi / 7=\pi / 3.5$ can indeed be considered as a two-dimensional flow. The two last curves of figure 15 show that amplitudes $A \leqslant \pi / 4$ allow the flow to stay 3D in the neighbourhood of the body. Once again, as an example, figure 14 shows such a flow, for $A=\pi / 5$.

The bifurcation can then be tracked between $A=2 \pi / 7$ and $A=\pi / 4$. In order to locate the critical value, five amplitudes $A=\pi / \alpha$ have been tested, $\alpha$ being 3.5, 3.75, 3.8, 3.9 and 4 . The result is plotted on figure 17 , and by means of this figure, the critical value $A_{0}$ is located in the interval $\pi / 3.8 \leqslant A_{0} \leqslant \pi / 3.9$ : figure 18 shows the transverse enstrophy for these two values. One could notice that this critical value is slightly depending on the computational domain, because at this amplitude a larger domain would contain a small amount of three-dimensional structures of vorticity. Indeed, one must keep in mind that we focus on the amount of threedimensionality in the (large) neighbourhood $r \leqslant(1+4 \pi) R$ of the body.

An open question is the dynamic of these three-dimensional structures. Indeed, they can either follow the potential stream and leave infinitely far away from the body, or, as suspected but not proved, reach a critical distance where three-dimensionality is stable. The computational cost required to prove this is currently by far out of reach.

To summarize on all these computations, one can plot the mean residual transverse enstrophy between $t_{1}=250$ and $t_{2}=300$, that is to say

$$
\begin{aligned}
\overline{Z^{\perp}}=\frac{1}{t_{2}-t_{1}} \int_{t_{1}}^{t_{2}} Z^{\perp}(t) \mathrm{d} t & =\frac{1}{2\left(t_{2}-t_{1}\right)} \int_{t_{1}}^{t_{2}}\left\|\omega_{x}(t)\right\|_{2}^{2}+\left\|\omega_{y}(t)\right\|_{2}^{2} \mathrm{~d} t \\
& =\frac{1}{2\left(t_{2}-t_{1}\right)} \int_{t_{1}}^{t_{2}} \int_{\Omega} \omega_{x}(\xi, t)^{2}+\omega_{y}(\xi, t)^{2} \mathrm{~d} \xi \mathrm{d} t
\end{aligned}
$$

with respect to the amplitude $A$. This curve, plotted on figure 18, gives the mean remaining amount of non-axial vorticity, and its behaviour when the rotation amplitude $A$ varies. This figure shows that this critical value makes a clear transition between $2 \mathrm{D}$ flows (to the right of the critical value) and 3D flows (to the left of the critical value).

\section{Mechanisms involved in the return to a two-dimensional flow}

From the numerical investigations performed above, one can identify two kinds of residual transverse vorticity (the present study is still performed at $R e=400$ ).

These two states of residual three-dimensionality are described as follows. When frequency is low (here at the natural frequency $S_{F}=S_{t}$ ), a small amount of three-dimensionality remains close to the body, where gradients of vorticity are the strongest. When frequency is higher (here at $S_{F}=2 S_{t}$ ), residual 3D structures are carried by the stream, and seem to loose any significant values, at least up to an hypothetic critical distance, which is in any case outside the present computational domain. Snapshots of these two cases are shown on figure 19, which exhibits a low level of transverse isovorticity. Note that these levels are low, thus these two flows are close to a two-dimensional state.

In this section, one can focus on the case $S_{F}=2 S_{t}$ (since the harmonic case remains slightly three-dimensional). The return to a two-dimensional flow has already been quantified above by means of the transverse enstrophy $Z^{\perp}$ (see section 5 and figure 11). 
Before quantifying the mechanism leading to a two-dimensional flow, one can describe it qualitatively. Indeed, one can guess in reading figure 20 that transverse eddies (non-spanwise structures of vortices) are carried down the stream by the spanwise von Kármán alleys. The strength of the von Kármán alleys becomes higher when the cylinder is rotating, because the vorticity created in the boundary layer is $\omega_{z}=-2 \pi R L V_{\text {slip }}$ and tends to give strength near the body.

In order to quantify this phenomenon, i.e. the fact that spanwise von Kármán alleys carry the transverse eddies downstream, one can define two quantities, still based on partial values of the enstrophy. They are the streamwise distribution of transverse vorticity

$$
F^{\perp}(r, t)=\int_{0}^{L} \int_{0}^{2 \pi} \omega_{x}^{2}(r, \theta, z)+\omega_{y}^{2}(r, \theta, z) r \mathrm{~d} \theta \mathrm{d} z
$$

and the streamwise distribution of spanwise vorticity

$$
F_{z}(r, t)=\int_{0}^{L} \int_{0}^{2 \pi} \omega_{z}^{2}(r, \theta, z) r \mathrm{~d} \theta \mathrm{d} z
$$

which satisfy

$$
\int_{R}^{R_{\infty}} F^{\perp}(r, t)+F_{z}(r, t) \mathrm{d} r=2 Z
$$

Indeed, isovalues of these quantities gives a good idea of the dynamics of transverse and spanwise eddies, providing a link between streamwise position and time. Moreover, a curve of low value of $F^{\perp}$ gives the evolution of the front of three-dimensionality, as plotted on figure 21 . This figure shows both the curves $F^{\perp}(r, t)=0.25$ (evolution of the front of three-dimensionality) and $F^{\perp}(r, t)=20$ (evolution of the front of strong transverse eddies).

In order to study quantitatively the evolution of the front of transverse eddies, it is interesting to compare their dynamics with spanwise von Kármán alleys dynamics. This comparison is provided by means of the isovalue $F_{z}(r, t)=5$, plotted on figure 22, which shows the evolution of the positions von Kármán alleys. Both curves $F_{z}(r, t)=5$ and $F^{\perp}(r, t)=0.25$ are plotted on the right picture of figure 22, on which it is rather clear that after $t=175$, the front of transverse eddies is locked on von Kármán alleys and is brought away downstream.

Consequently, in this section, from now on, one aims at proving the following assertion : the three-dimensional instabilities decrease in strength and are brought away in the stream when rotation frequency and amplitude are high enough. They finally disappear due to this transport phenomenon. The fact that they decrease in strength and eventually disappear is already proved because

$$
F^{\perp}(r, t) \underset{t \rightarrow \infty}{\longrightarrow} 0
$$

for any $r$, as shown on figure 21.

In order to quantify the transport phenomenon, one takes out the von Kármán alley on which the front of instabilities seems to lock on, and one defines the front location of spanwise eddies

$$
X_{z}(t)=\operatorname{argmin}\left\{r \text { such as } F_{z}(r, t)=5\right\}
$$

and the front location of transverse eddies

$$
X^{\perp}(t)=\operatorname{argmin}\left\{r \text { such as } F^{\perp}(r, t)=0.25\right\}
$$

as shown on figure 23 .

These quantities are useful in the present case, because the assertion above claiming that instabilities are captured by von Kármán alleys is equivalent to have a linear correlation between $X_{z}$ 
and $X^{\perp}$ over time. Figure 24 shows $X^{\perp}$ with respect to $X_{z}$, and the dependency is clearly linear when $r>7 R$. One eventually gets a correlation coefficient of $99.99 \%$. The assertion is then proved to be valid, and the mechanism making the instabilities disappear in the wake is identified. To conclude the present analysis, a comment on the domain of return to a two-dimensional flow is needed. An hypothetic critical distance from the body where the flow stays three-dimensional has been mentioned above. Indeed, the enstrophy distribution, as a function of the distance from the body, is exponentially decreasing, thus reaching a value for which transverse vortex structures are stable. Such a stability analysis recquires very large domains with a control of cells' aspect ratio, which is not currently technically possible.

\section{Conclusions}

A lagrangian numerical method has been built to solve the fully non-linear three-dimensional Navier-Stokes equations. The stability due to the implicit resolution of transport terms allows the use of large time steps. This means one can compute and study the asymptotic behaviour in time of three-dimensional flows. The numerical scheme has been validated on both two-dimensional and three-dimensional diagnostics of flows behind a steady cylinder, and on two-dimensional flows past a rotating cylinder. The results obtained are consistent with some of the existing literature, numerically as well as experimentally.

It has been shown, at $R e=400$, that in a large neighbourhood of the body, the flow comes back to its nominal two-dimensional state all over the domain when the amplitude and frequency are high enough (presently $A=\pi / 2$ and $S_{F} / S_{t}=2$ ). In this case, a substantial drop of the drag coefficient is exhibited, though not as dramatic as for experimentations by Tokumaru \& Dimotakis (1991) performed at $R e=15000$.

When the forced and natural Strouhal numbers coincide, a small amount of three-dimensionality remains very close to the body (figures 13 and 19). The present results also validate 2D numerical simulations of previous works, depending on the amplitude values.

A parametric study of the topology of flow has been then performed at the first super-harmonic frequency $S_{F} / S_{t}=2$. A bifurcation diagram (figure 18) shows a clear transition between the 2D and 3D resulting flows. The different kinds of residual three-dimensionality have been exhibited and the mechanism leading to a two-dimensional state has been identified. This behaviour has not previously been exhibited numerically and seems to make a quite new contribution to the physics of the problem.

In summary, it has been shown by means of a parametric study that the wake generated by a translating and rotationally oscillating circular cylinder can lead either to a $2 \mathrm{D}$ or a $3 \mathrm{D}$ flow for the same Reynolds number, depending of on the rotation parameters.

The author would like to acknowledge the invaluable help of Georges-Henri Cottet (University J. Fourier, Grenoble, France) and Petros Koumoutsakos (Institute of Computational Science, ETH Zürich, Switzerland), for their contributions in the preparation of this paper. The author would also like to thank Michele Milano (California Institute of Technology) for his helpful suggestions is the early development of this work. The computational resources have been provided by the joint CEA-UJF CIMENT-MIRAGE project, by the CEA-CENG (cluster IXIA), by the Department of Mathematics and Modelisation (INSA Toulouse, France) and CALMIP (CICT, Toulouse, France). 
- Figures -

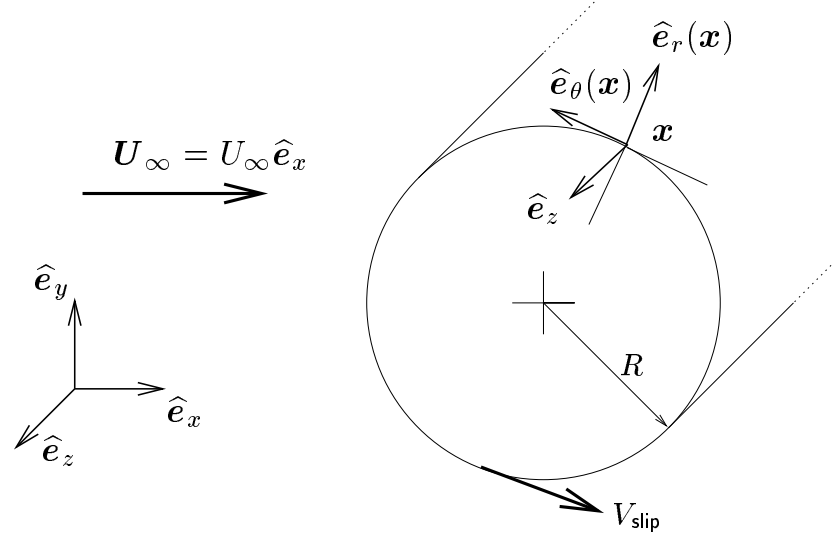

FIGURE 1. Cartesian and cylindrical basis.
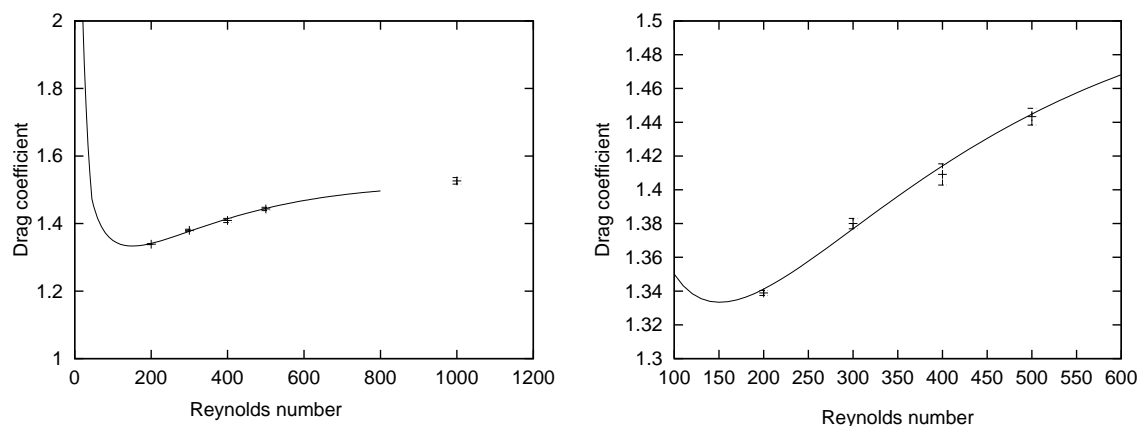

FIGURE 2. Comparison of 2D drag coefficients between Henderson's benchmark values (-) and table 1 with $90 \%$ confidence intervals. Right picture is a zoom of left picture. 

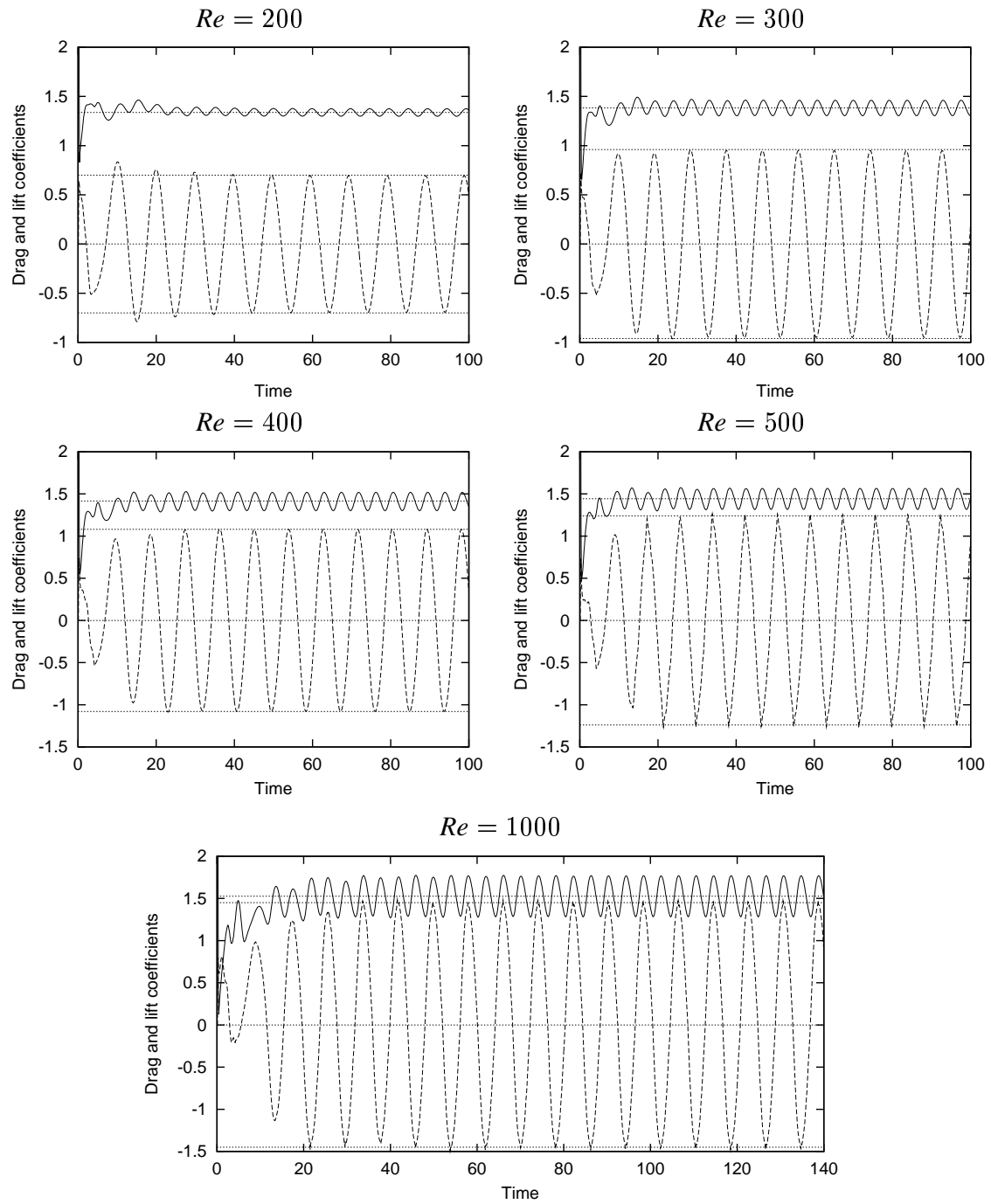

FIGURE 3. Drag and lift coefficients at $R e=200,300,400,500$ and 1000. $C_{D},--C_{L}, \cdots$ values from table 1.
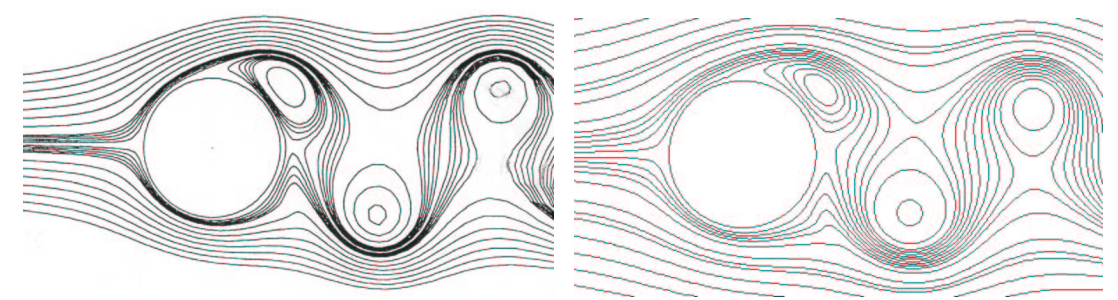

FIGURE 4. Streamlines at $R e=500$ for $A=3 / \pi$ and $S_{F}=1 / 3 \simeq 1.45 S_{t}$, obtained by Dennis et al. (2000) (to the left), and present work (to the right). Left picture kindly provided by S.C.R. Dennis. 
18

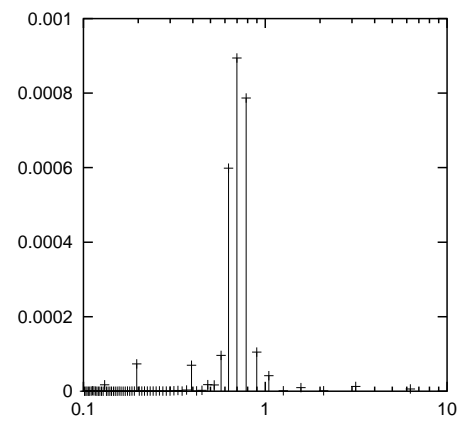

Wavelength $\lambda_{z} / D$
P. Poncet
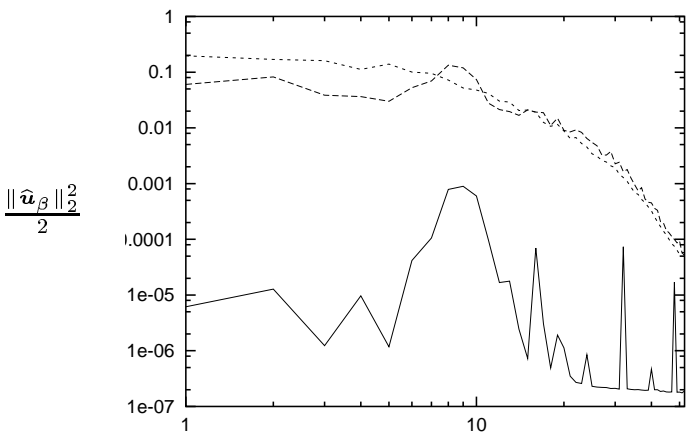

Wave number $\beta$

FIGURE 5. Axial energy spectrum at $R e=400$. Left picture : Energy spectrum with respect to $\lambda_{z} / D$ and its resonance at $\lambda_{z} / D \simeq 0.73$, at $t=60$ during the transient between $2 \mathrm{D}$ and 3D. Right picture : Energy spectra with respect to the modes $\beta$ at $t=60(-), t=100(--)$ and $t=150(--)$.

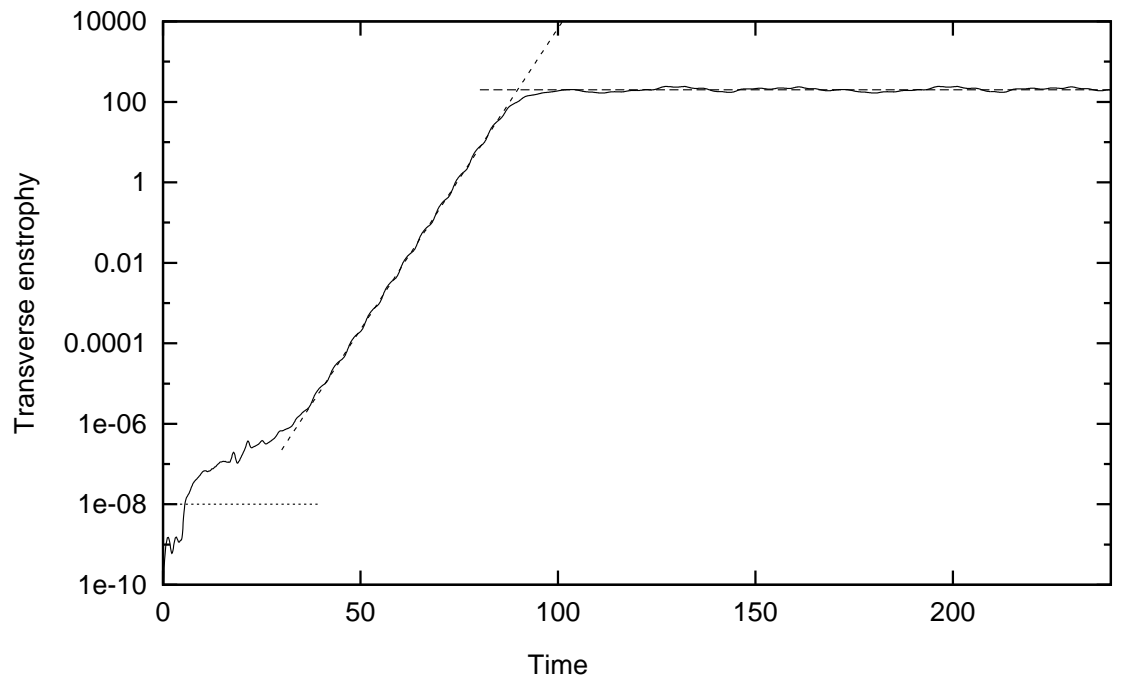

Figure 6. Development of transverse enstrophy $Z^{\perp}=\left(\left\|\omega_{x}\right\|_{2}^{2}+\left\|\omega_{y}\right\|_{2}^{2}\right) / 2$ at $R e=400$. Dashed lines show the constant value 200 and the exponential $a e^{t / 2.9}$ with $a=7.4110^{-12}$.
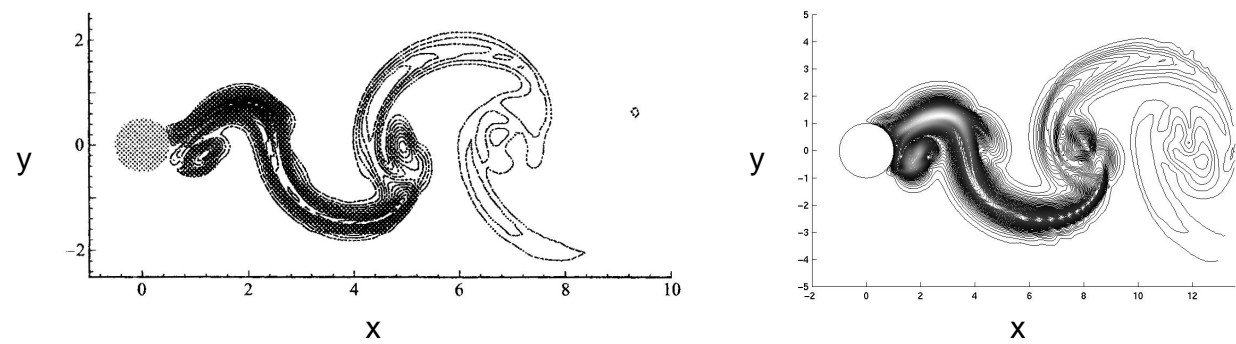

FIGURE 7. Isovalues of spectral profile at main growth mode. Left picture $: \mathfrak{P}_{0.82}$ of Barkley $\&$ Henderson (1996) at $R e=260$. Right Picture $: \mathfrak{P}_{0.79}$ of present work at $R e=400$. 


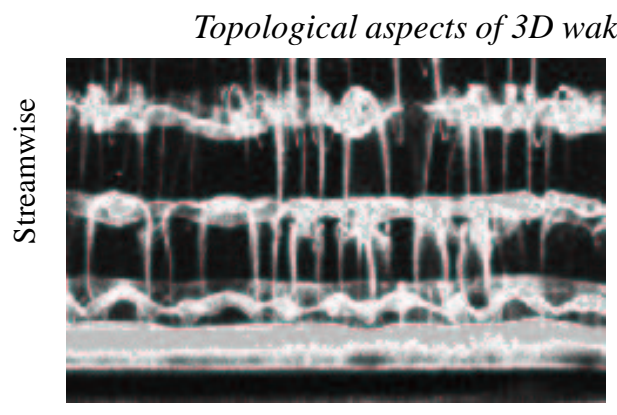

spanwise

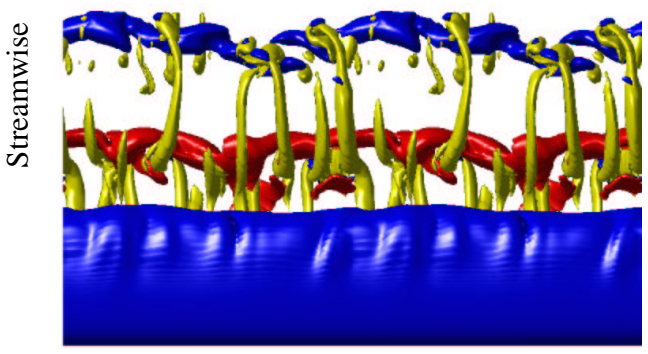

spanwise

FIGURE 8. Visual comparison of three-dimensional structures of vorticity between experimentation (left picture, Williamson (1996) at $R e=270$ ) and present work (right picture, at $R e=400$, doubled spanwise by periodicity, $t=160)$.
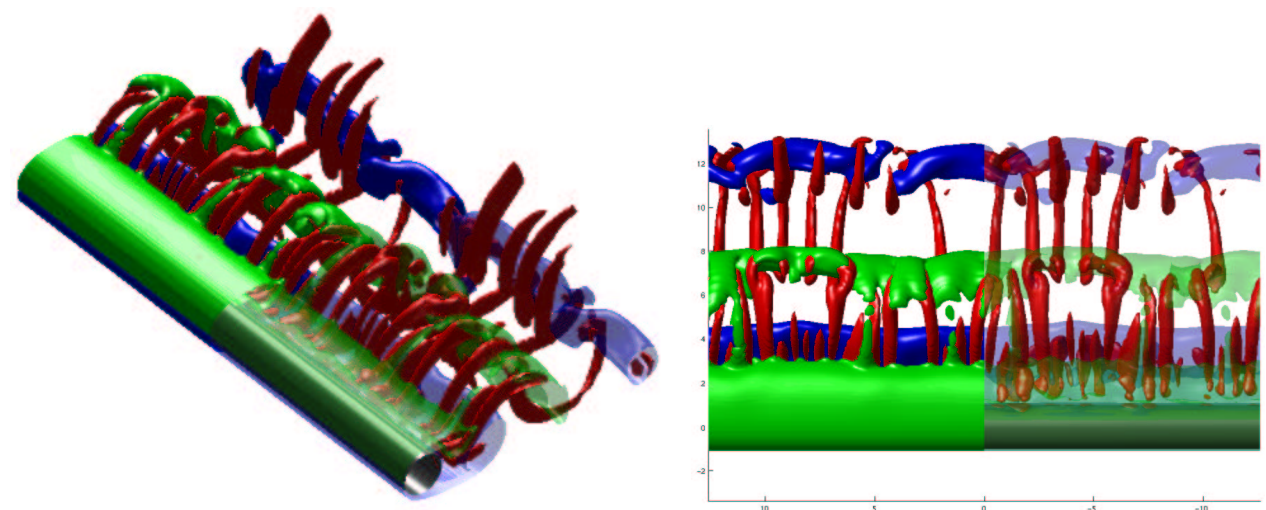

FIGURE 9. Three-dimensional structures of vorticity, present work at $R e=300$, doubled spanwise by periodicity $(t=350)$, with transparency of axial vorticity on the right part.

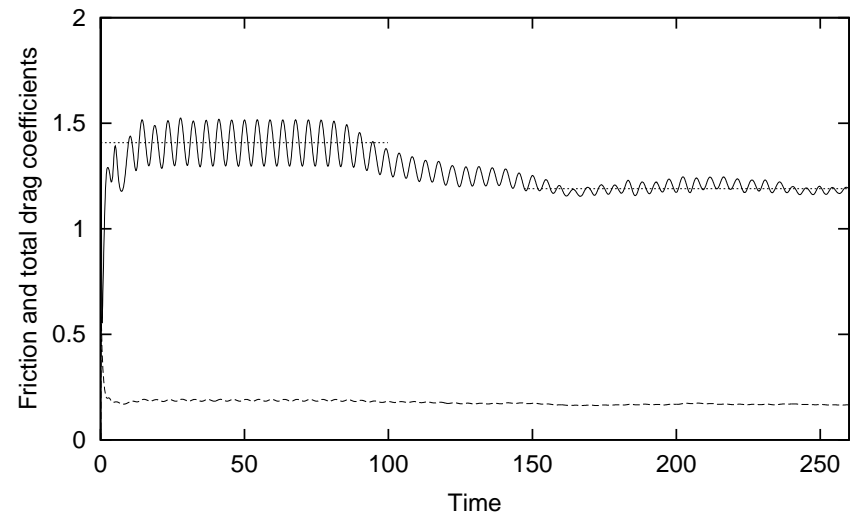

FIGURE 10. Effect of three-dimensionality on the drag coefficient $C_{D}$ at $R e=400$. The friction coefficient $C_{D F}$ is plotted with dashed lines. Dotted lines show values at 1.41 and 1.19. 


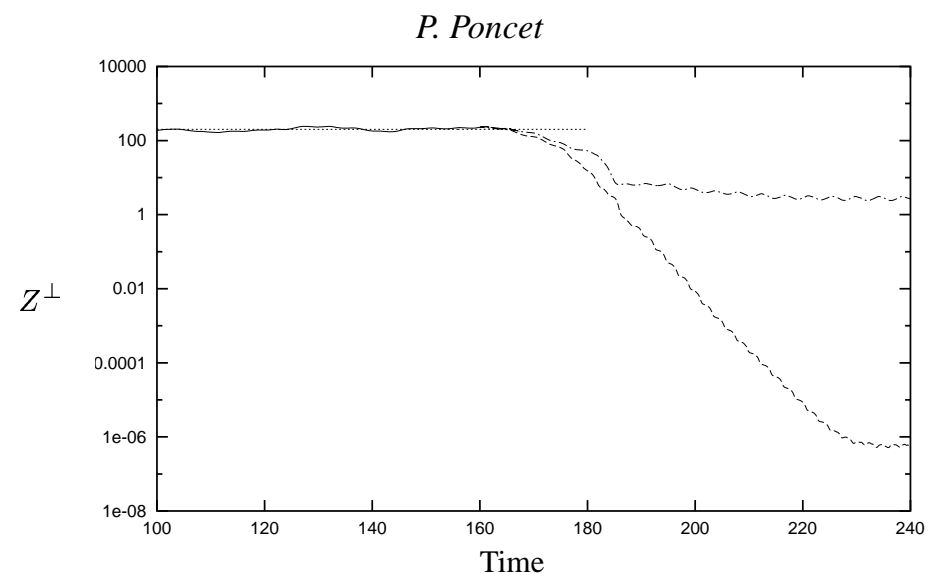

FIGURE 11. Transverse enstrophy $Z^{\perp}$ at $R e=400$ with respect to time, for a rotation amplitude $A=\pi / 2 .--: S_{F}=2 S_{t}, \cdot-\cdot: S_{F}=S_{t}$.

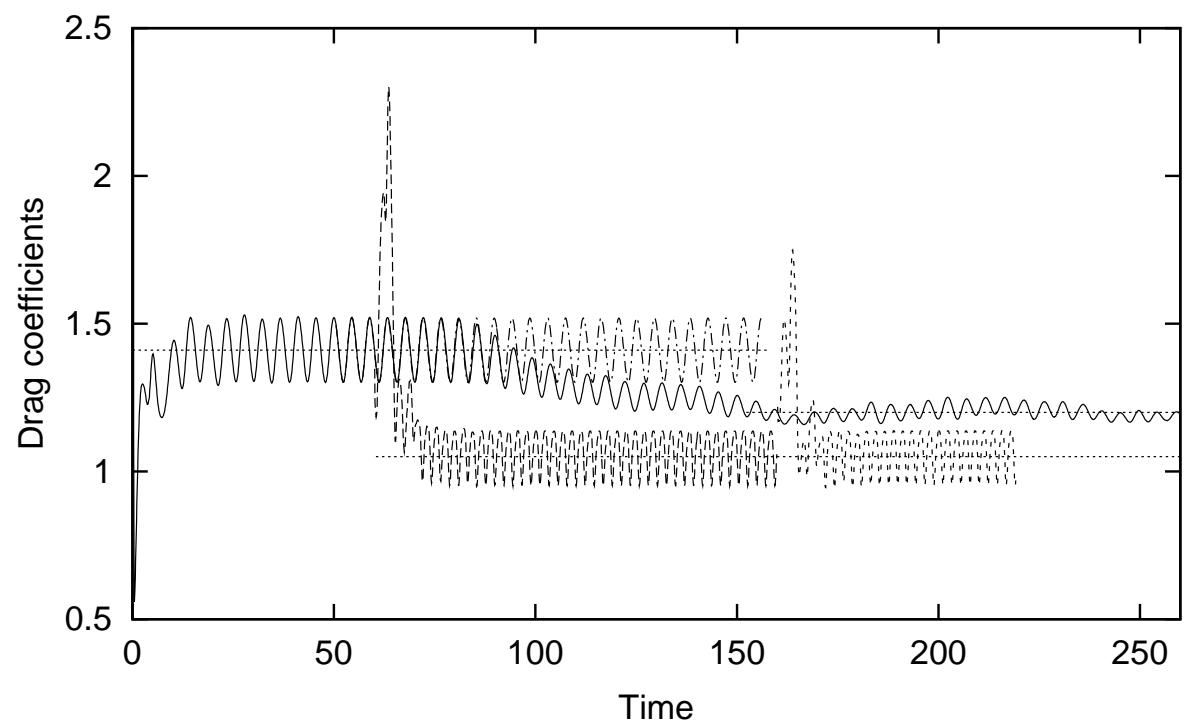

FIGURE 12. Effect of body rotation on the drag coefficient $C_{D}$ at $R e=400$, with $S_{F}=2 S_{t}$ and $A=\pi / 2$. - : Steady and 3D, $\cdot-\cdot:$ Steady and 2D, $--:$ Rotation 2D (dashed line to the left), - - : : Rotation 3D (dashed line to the right). Dotted lines show values at 1.05, 1.19 and 1.41. 

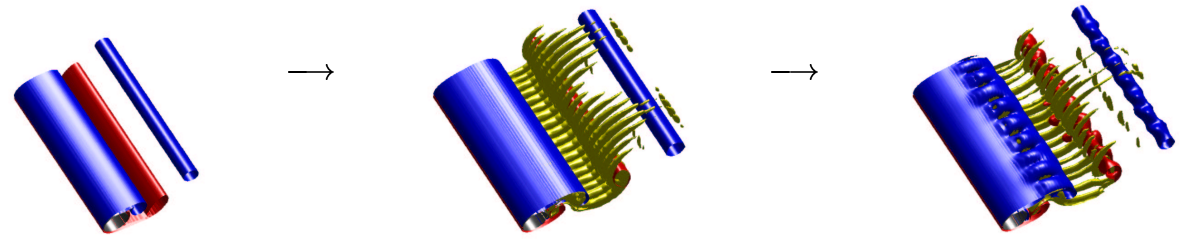

$t=10$

$$
\text { Signature of instability at }
$$
$t=50, \omega^{\perp}=3.510^{-4}$

$$
t=100, \omega^{\perp}=1.2
$$

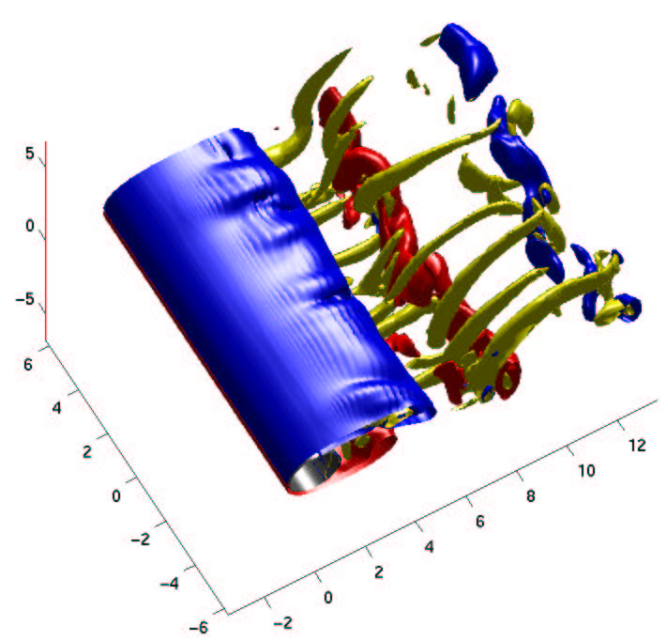

$$
\swarrow \searrow t=160, \omega^{\perp}=1.5 \text {, rotation begins }
$$
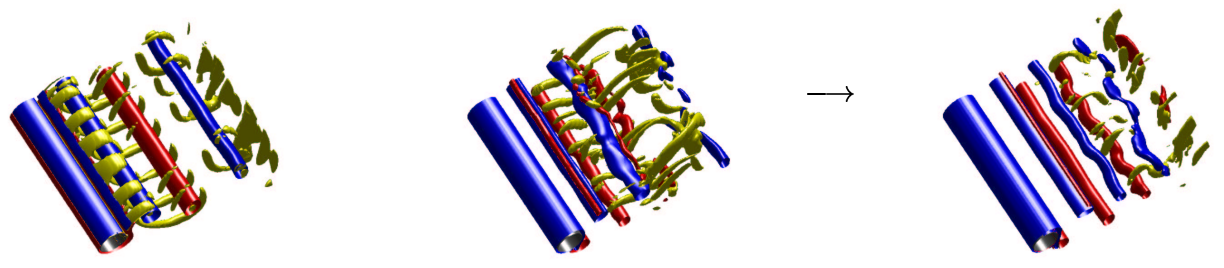

$S_{F}=S_{t}, t=185$

$S_{F}=2 S_{t}, t=170, \omega^{\perp}=1.2$

$S_{F}=2 S_{t}, t=175, \omega^{\perp}=1.2$

$\downarrow$

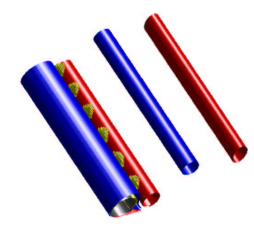

$S_{F}=S_{t}, t=240$

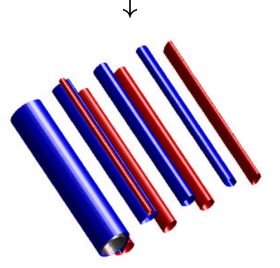

$S_{F}=2 S_{t}, t=240$

FIGURE 13. Effect of half a full rotation on the shape of the flow at $R e=400$. Surfaces of spanwise isovorticity $\omega_{z}= \pm 1.2$ and transverse vorticity $\omega^{\perp}=0.25$ unless written otherwise, for frequencies $S_{F}=S_{t}$ and $S_{F}=2 S_{t}$. 


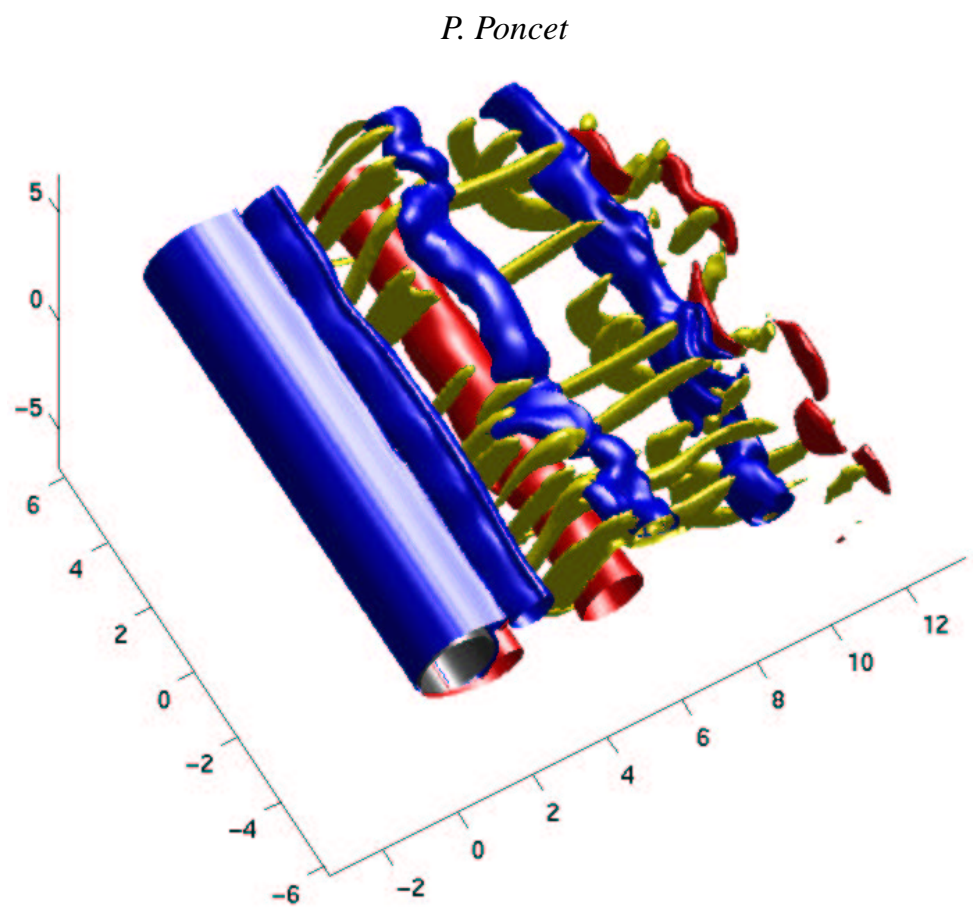

FIGURE 14. Isovorticity surface at $t=255, R e=400, S_{F}=2 S_{t}$ and $A=\pi / 5$.

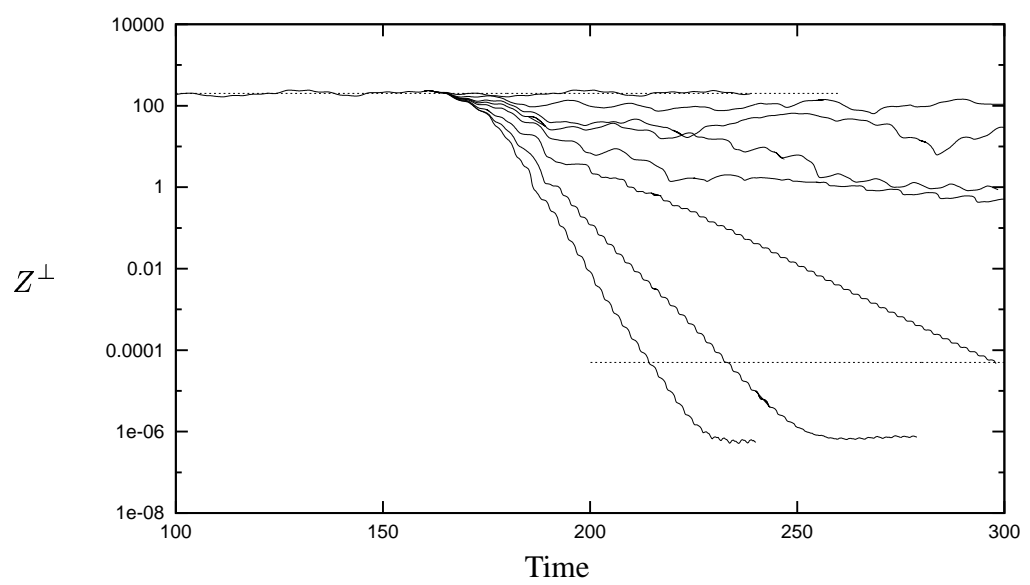

FIGURE 15. Transverse enstrophy $Z^{\perp}$ at $R e=400$ with respect to time, for several rotation amplitudes, in logarithmic scale. From bottom to top : $A=\pi / 2, A=2 \pi / 5, A=\pi / 3, A=2 \pi / 7, A=\pi / 3.75$, $A=\pi / 4, A=\pi / 5$ and $A=0$ (steady). 


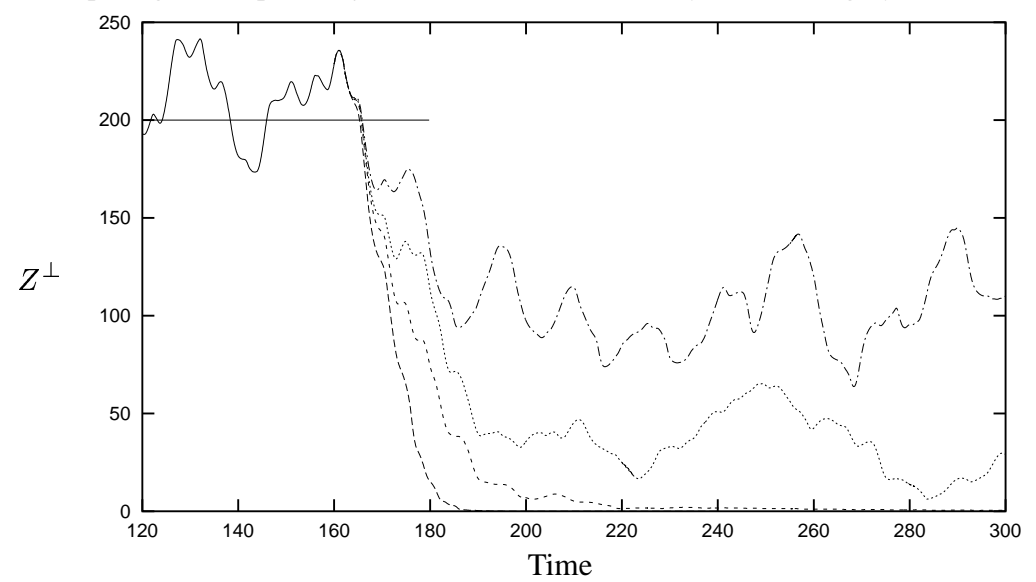

FIGURE 16. Transverse enstrophy $Z^{\perp}$ at $R e=400$ with respect to time, for several rotation amplitudes. From bottom to top : $A=\pi / 2, A=\pi / 3.5, A=\pi / 4$ and $A=\pi / 5$.
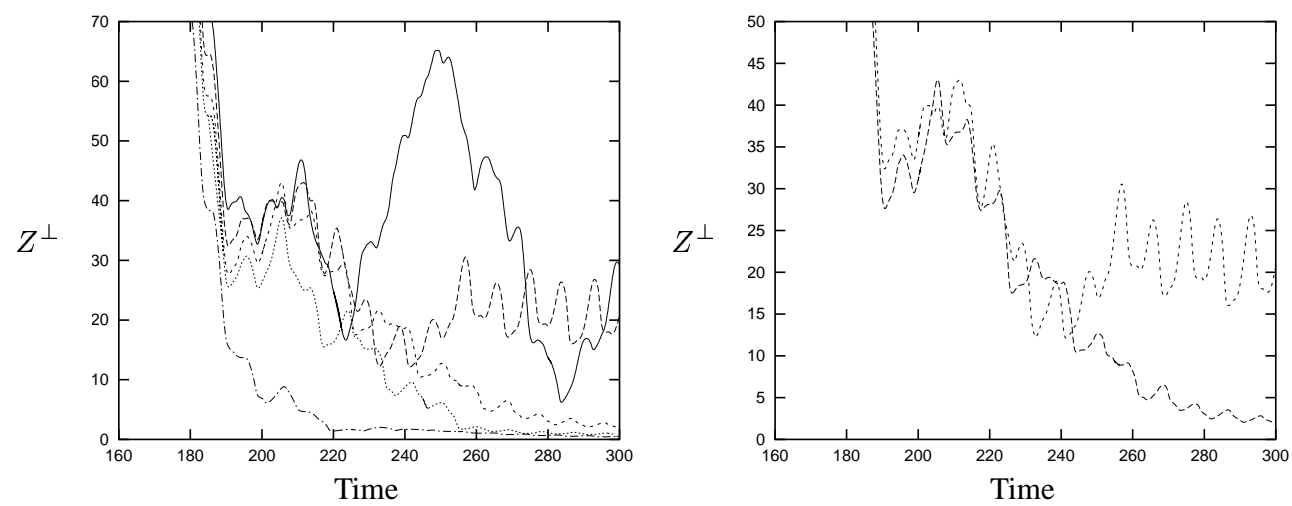

FIGURE 17. Transverse enstrophy $Z^{\perp}$ at $R e=400$ with respect to time, for several rotation amplitudes close to the bifurcation value. Left picture, from bottom to top : $A=10 \pi / 35, A=10 \pi / 37.5$, $A=10 \pi / 38, A=10 \pi / 39$ and $A=10 \pi / 40$. Right picture : only amplitudes $A=\pi / 3.8$ and $A=\pi / 3.9$.

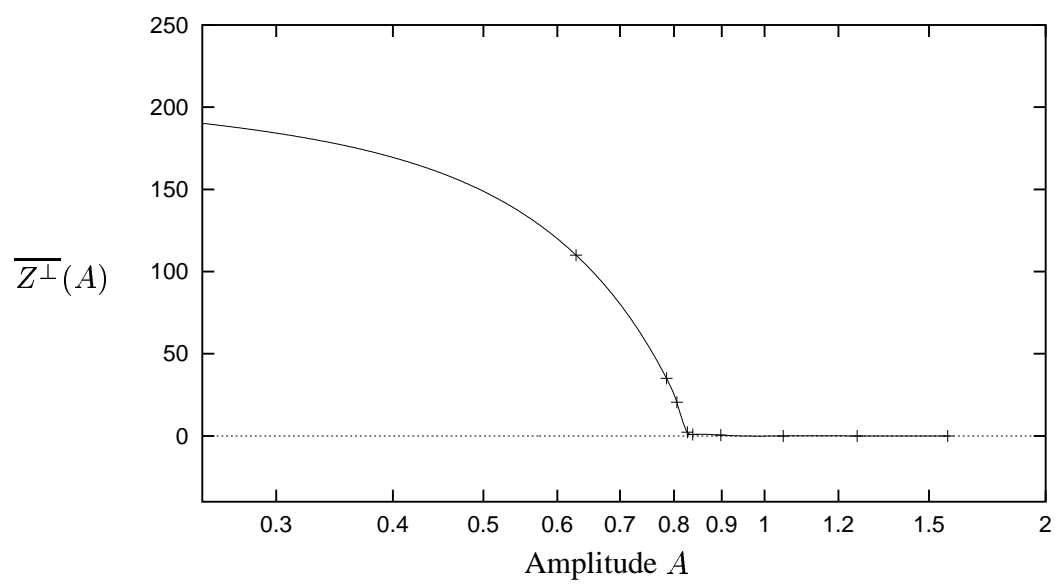

FIGURE 18. Mean residual transverse enstrophy $\overline{Z^{\perp}}$ (defined in text) with respect to the rotation amplitude $A$, at $R e=400, S_{F}=2 S_{t}$. : Computations, - : Curve linking the points by interpolation. 

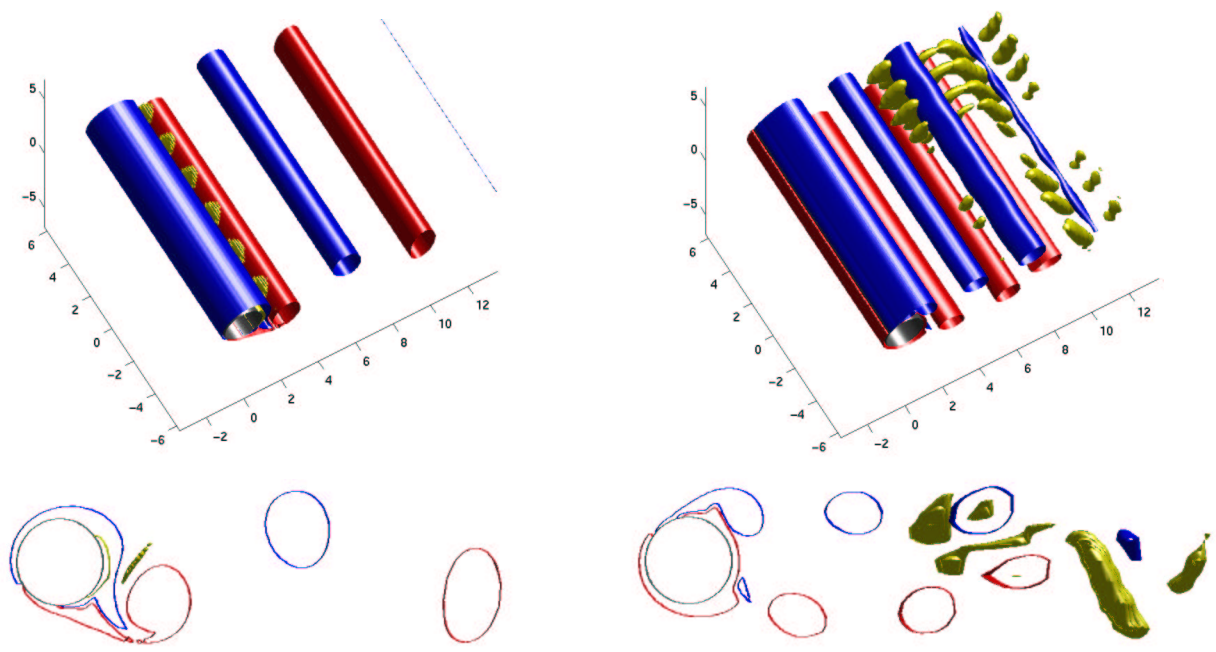

$S_{F}=S_{t}$ and $A=\pi / 2, \omega^{\perp}=0.25$

$S_{F}=2 S_{t}$ and $A=2 \pi / 7, \omega^{\perp}=0.1$ FIGURE 19. Localisation of residual transverse vorticity, at $t=280$.
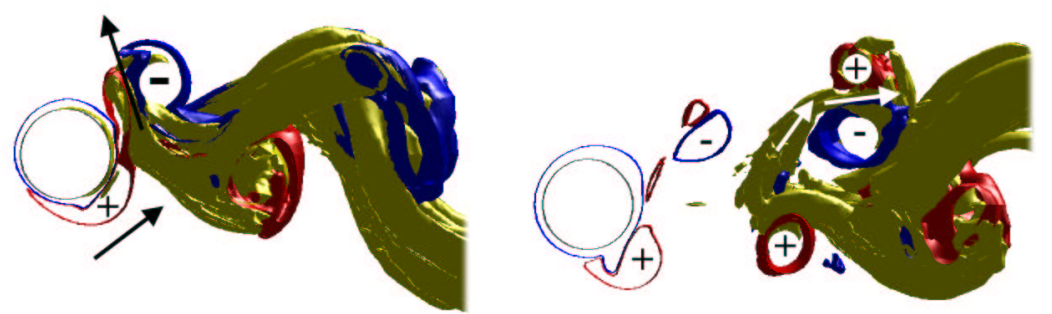

$$
t=165
$$

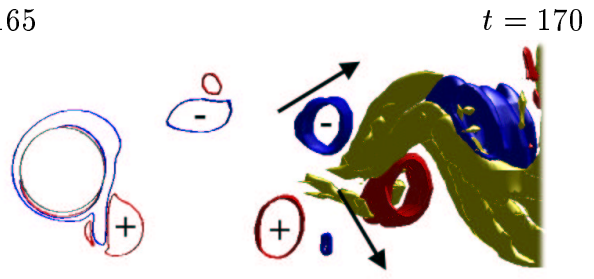

$$
t=175
$$

Figure 20. Motion of transverse eddies, $R e=400, A=\pi / 2$ and $S_{F}=2 S_{t}$. 

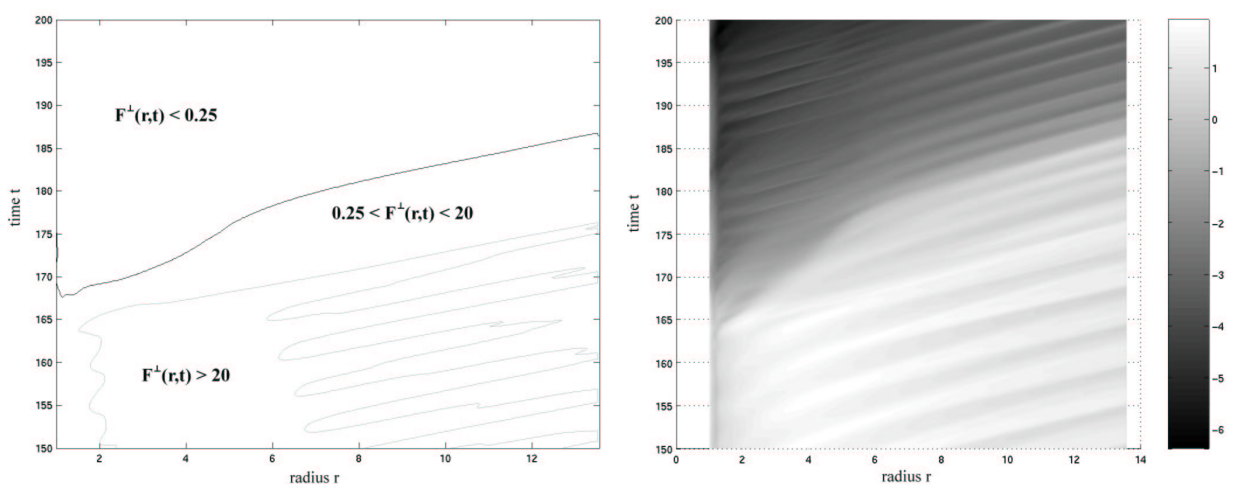

FIGURE 21. Dynamics of streamwise distribution of transverse vorticity $F^{\perp}(r, t)$ at levels 0.25 and 20, for $R e=400, A=\pi / 2$ and $S_{F}=2 S_{t}$, rotation activated at $t=160$. Colour bar : $\log _{10} F^{\perp}(r, t)$.
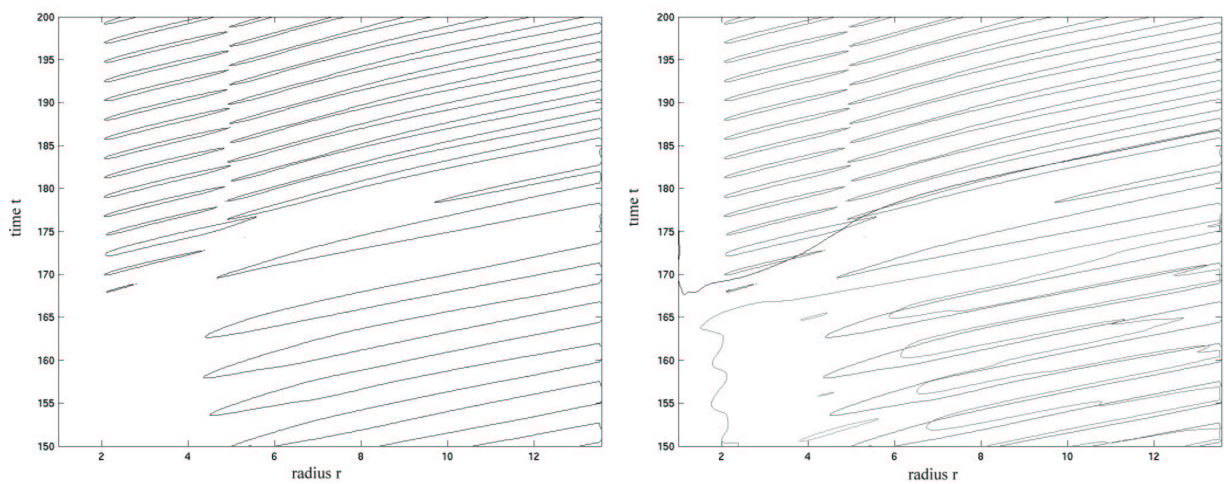

FIGURE 22. Dynamics of streamwise distribution of spanwise vorticity $F_{z}(r, t)$. The isovalue $F_{z}(r, t)=5$ is plotted on left picture. On right picture, the streamwise distribution of vorticity $F^{\perp}(r, t)$ (from figure 21 ) is added. Same parameters as fig. 21.

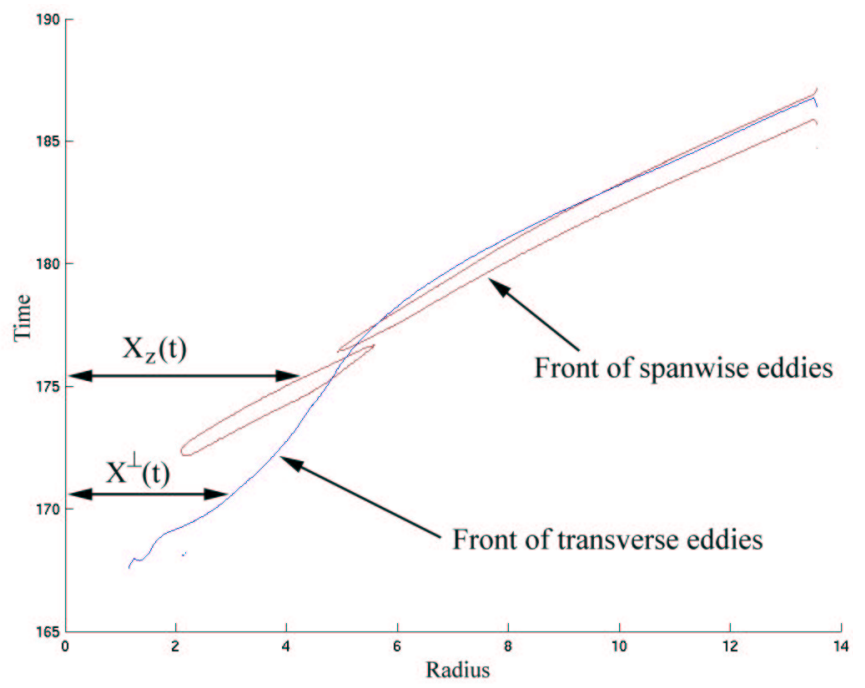

FIGURE 23. Definition of front location of spanwise $\left(X_{z}(t)\right)$ and transverse $\left(X^{\perp}(t)\right)$ eddies, respectively at level 5 and 0.25 (same parameters as fig. 21). 

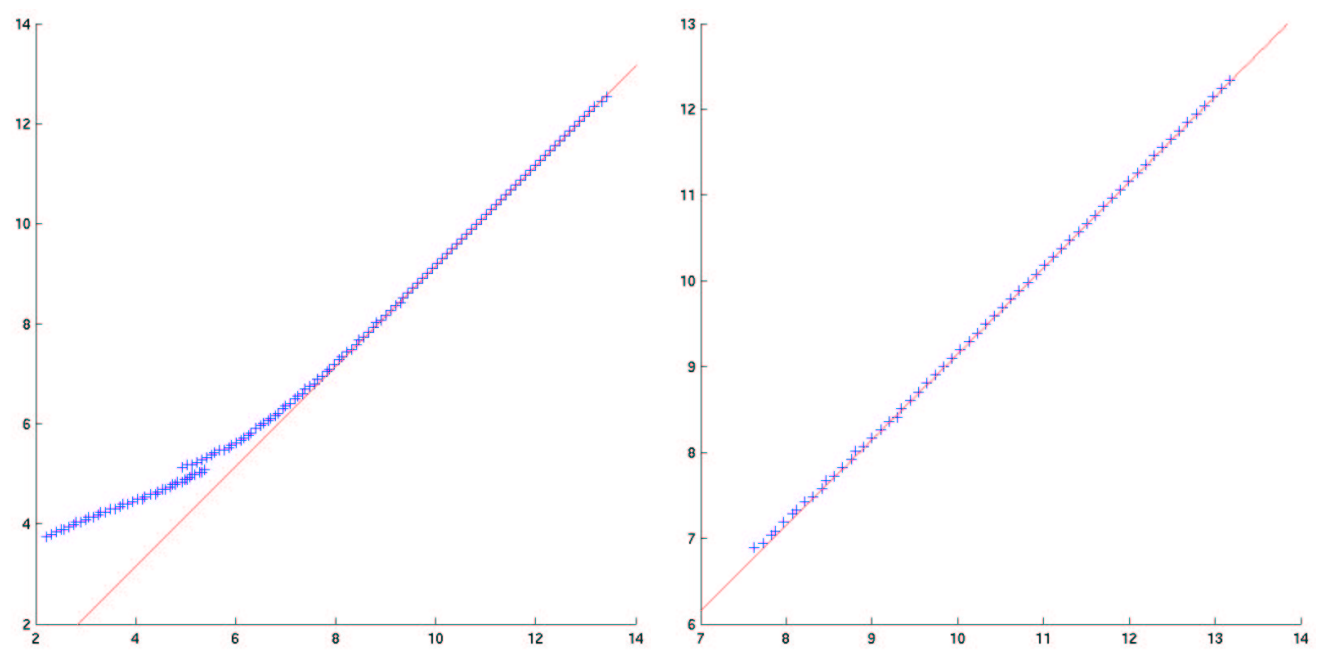

FIGURE 24. Front locations $X^{\perp}(t)$ with respect to $X_{z}(t)$ (right picture is a zoom of left picture, parameters are the same as for figure 21). 


\section{REFERENCES}

BADR, H. M. \& DENNIS, S. C. R. 1985 Time-dependent viscous flow past an impulsively started rotating and translating circular cylinder. J. Fluid Mech. 158, 447-488.

Badr, H. M., Coutanceau, M., Dennis, S. C. R. \& MÉnard, C. 1990 Unsteady flow past a rotating circular cylinder at Reynolds numbers $10^{3}$ and $10^{4}$. J. Fluid Mech. 158, 447-488.

BAEK, S. J. \& SUNG, H. J. 1998 Numerical simulations of the flow behind a rotary oscillating circular cylinder. Phys. Fluids 10, 869-876.

BARKLEY, D. \& Henderson, R. D. 1996 Three-dimensional Floquet stability analysis of the wake of a circular cylinder. J. Fluid Mech. 322, 215-241.

BEARMAn, P. 1997 Bluff body hydrodynamics. In Twenty-first symposium on naval hydrodynamics, Trondheim, Norway.

BeAudan, P. \& Moin, P. 1994 Numerical experiments on the flow past a circular cylinders at sub-critical Reynolds numbers. Tech. rep. TF-62, Stanford.

BlackBurn, H. M. \& Henderson, R. D. 1999 A study of two-dimensional flow past an oscllating cylinder. J. Fluid Mech. 385, 255-286.

ChAng, C. C. \& Chern, R. L. 1991 Vortex shedding from an impulsively started rotating translating circular cylinder. J. Fluid Mech. 233, 265-298.

Chen, Y. M., OU, Y. R. \& PeARlstein, A. J. 1993 Development of the wake behind a circular cylinder impulsively started into rotatory and rectilinear motion. J. Fluid Mech. 253, 449-484.

CHOU, M. H. 1997 Synchronization of vortex shedding from a cylinder under rotary oscillation. Comput. Fluids 26, 755-774.

Cottet, G.-H. 1996 On three-dimensional vortex schemes. In ASME Fluids Conf., San Diego.

Cottet, G.-H. \& Koumoutsakos, P. D. 2000 Vortex methods, theory and practice. Cambridge University Press.

Cottet, G.-H., Michaux, B., Ossia, S. \& Vanderlinden, G. 2002 A comparison of spectral and vortex methods in three-dimensional incompressible flows. J. Comp. Phys. 175, 702.

Cottet, G.-H. \& Poncet, P. 2002 Particle methods for Direct Numerical Simulations of threedimensional wakes. J. Turbulence, 3(038), 1-9.

Cottet, G.-H. \& Poncet, P. 2003 Advances in Direct Numerical Simulations of three-dimensional wallbounded flows by Particle in Cell methods. J. Comp. Phys. 193, 136-158 (2003).

Cottet, G.-H. \& Poncet, P. 2004 New results in the simulation and control of three-dimensional cylinder wakes. Comput. Fluids 33, 687-713.

Coutanceau, M. \& MÉnARd, C. 1985 Influence of rotation on the near-wake behind an impulsively started circular cylinder. J. Fluid Mech. 253, 449-484.

Degond, P. \& MAS-GALLIC, S. 1989 The weighted particle method for convection-diffusion equations. Math. Comput. 53, 485-526.

Dennis, S. C. R., NGUYen, P. \& KocabiyiK, S. 2000 The flow induced by a rotationally oscillating and translating circular cylinder. J. Fluid Mech. 385, 255-286.

DÜTCH, H., DuRST, F., BECKER, S. \& LiEnhART, H. 1998 Low-Reynolds-number flow around an oscillating circular cylinder at low Keulegan-Carpenter numbers. J. Fluid Mech. 360, 249-271.

Friedmann, A. 1964, Partial differential equations of parabolic type. Prenctice-Hall.

Govardhan, R. \& Williamson, C. H. K. 2000 Modes of vortex formation and frequency response of a freely vibrating cylinder. J. Fluid Mech., 420, 85-130.

He, J.-W., Glowinski, R., Metcalfe, R., Nordlander, A. \& Periaux, J. 2000 Active Control and drag optimization for flow past a circular cylinder, I. Oscillatory cylinder rotation J. Comp. Phys., $163,83-117$.

Henderson, R. D. 1995 Adaptative spectral element methods for turbulence and transition. Tech. rep., California Institute of Technology.

Henderson, R. D. 1995 Details of the drag curve near the onset of vortex shedding. Phys. Fluids 7(9), 2102-2104.

Henderson, R. D. 1997 Nonlinear dynamics and patterns in turbulent wake transition. J. Fluid Mech. 352, 65-112.

Huerre, P. \& Monkewitz, P. A. 1990 Local and global instabilities in spatially developing flows. Ann. Rev. Fluid Mech. 22, 473-537.

JordAn, S. K. \& Fromm, J. E. 1972 Oscillatory drag, lift and torque on a circular cylinder in a uniform flow. Phys. Fluids 15:371-376. 
JORDAN, S. 1997 Large-Eddy simulation of the vortical motion resulting from flow over bluff bodies. In Twenty-first symposium on naval hydrodynamics, Trondheim, Norway.

KAng, S., ChOI, H. \& LeE, S. 1999 Laminar flow past a rotating cylinder Phys. Fluids. 11(11), 33123321.

Karniadakis, G. \& Triantafyllou, G. 1992 Three dimensional dynamics and transition to turbulence in the wake of bluff objects. J. Fluid Mech., 238,1-30.

Koumoutsakos, P. D., Leonard, A. \& PePIN, F. 1994 Boundary conditions for viscous vortex methods. J. Comp. Phys. 113, 52.

Koumoutsakos, P. D. \& LEONARD, A. 1995 High-resolution simulations of the flow around an impulsively started cylinder using vortex methods. J. Fluid Mech. 296, 1-38.

Kravchenko, A. G., Moin, P. \& Shariff, K. 1999 B-Spline method and zonal grids for simulations of complex turbulent flows. J. Comp. Phys. 151:757-789.

LINDSAY, K. \& KRASNY, R. 2001 A particle method and adaptive treecode for vortex sheet motion in 3-D flow. J. Comp. Phys. 172, 879-907.

LU, X. Y. \& SATO, J. 1996 A numerical study of flow past a rotationally oscillating circular cylinder. J. Fluids Struct. 10, 829-849.

Milano, M., Giannakopoulos, X. \& Koumoutsakos, P. D. 2000 Evolving strategies for active flow control. In Congress of Evolutionary Computations, San Diego.

Milano, M. \& Koumoutsakos, P. D. 2002 A clustering genetic algorithm for cylinder drag optimization. J. Comp. Phys. 175, 79-107.

Mittal, R. \& BALACHANDAR, S. 1995 Effects of three-dimensionality on the lift and drag of nominally two-dimensional flows. Phys. Fluid. 7, 1841-1865.

MittAL, R. 1996 Progress on LES of flow past a circular cylinder. Annual Research Briefs. Center for Turbulence Research.

Monaghan, J. J. 1985 Extrapolating B-Splines for interpolation. J. Comp. Phys. 60, 253-262.

OUld-S Ahili, M. L., COTTET, G.-H. \& El HAMraOuI, M. 2000 Blending finite-differencies and vortex methods for incompressible flow computations. SIAM J. Sci. Comput. 22, 1655-1674.

PERSILlon, H. \& BRAZA, M. 1998 Physical analysis of the transition to turbulence in the wake of a circular cylinder by three-dimensional Navier-Stokes simulation. J. Fluid Mech. 365, 23-88.

Ploumhans, P. \& Winckelmans, G. S. 2000 Vortex Methods for High-Resolution Simulations of Viscous Flow Past Bluff Bodies of General Geometry. J. Comp. Phys. 165, 354-406.

PONCET, P. 2002 Vanishing of mode B in the wake behind a rotating circular cylinder. Phys. Fluids. 14(6), 2021-2024.

Poncet, P. \& CotTet, G-H. 2003 Open-loop control of three-dimensional wakes. Second MIT conference. Boston, USA, 2348-2351.

Poncet, P. \& Koumoutsakos, P. 2004 Vortex Shedding Reduction in 3D Wakes using Belt Actuators and Genetic Algorithms. Accepted for Conference of International Society of Offshore and Polar Engineers ISOPE 2004.

Strickland, J. H., Gritzo, L. A., BAty, R. S. \& Homicz, G. F. 1999 Fast multipole solvers for three-dimensional radiation and fluid flow problems. In Third international workshop on vortex flows and related numerical methods, ESAIM Proceedings, Vol. 7.

SÜMER, B. M. \& Fredsøe, J. 1997 Hydrodynamics around Cylindrical Structures. World Scientific.

Thompson, M., Hourigan, K. \& Sheridan, J. 1994 Three-dimensional instabilities in the cylinder wake. In Intl. Colloq. Jets, Wakes and Shear Layer, Victoria, Australia.

Thompson, M., Hourigan, K. \& Sheridan, J. 1996 Three-dimensional instabilities in the wake of a circular cylinder. Exp. Therm. Fluid Sci., 12, 190-196.

TANEDA, S. 1991 Visual observations of the flow past a circular cylinder performing rotary oscillation. J. Phys. Soc. Japan 45, 1038-1043.

TOKUMARU, P. \& Dimotakis, P. 1991 Rotary oscillation control of a cylinder wake. J. Fluid Mech. 224, 77-90.

Tomboulides, A., Triantafyllou, G. \& Karniadakis, G. 1992 A new mechanism of period doubling in free shear flows. Phys. Fluids A, 4, 1329-1332.

Unal, M. F. \& Rockwell, D. 1988 On vortex formation from a cylinder. Part I, the initial stability. J. Fluid Mech. 190, 491-512.

UtNES, T. 1997 Two-grid finite element formulations of the incompressible Navier-Stokes equations. Comm. Numer. Meth. Eng. 13, 675-684. 
Williamson, C. H. K. 1988 The existence of two stages in the transition to three-dimensionality of a circular cylinder. Phys. Fluid. 31, 3165-3168.

Williamson, C. H. K. 1996 Three-dimensional wake behind a cylinder. J. Fluid Mech. 328.

WIESELSBERGER, C. 1922 New data on the laws of fluid resistance. NACA Tech. rep. TN-84. 